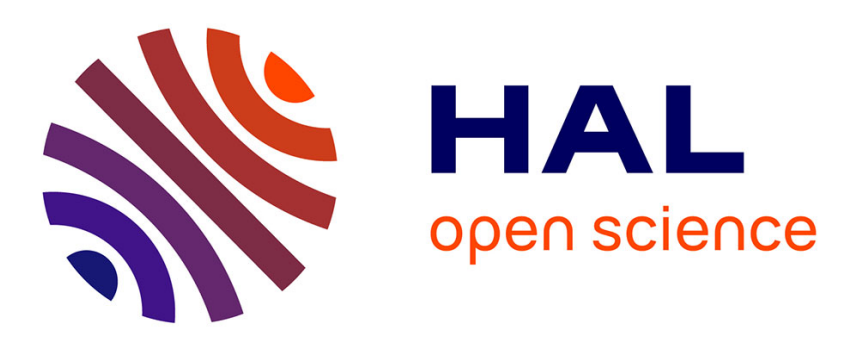

\title{
A comparison between two-scale asymptotic expansions and Bloch wave expansions for the homogenization of periodic structures
}

Grégoire Allaire, Marc Briane, Muthusamy Vanninathan

\section{To cite this version:}

Grégoire Allaire, Marc Briane, Muthusamy Vanninathan. A comparison between two-scale asymptotic expansions and Bloch wave expansions for the homogenization of periodic structures. SeMA Journal: Boletin de la Sociedad Española de Matemática Aplicada, 2016, 73 (3), pp.237-259. 10.1007/s40324016-0067-z . hal-01215580v2

\section{HAL Id: hal-01215580 \\ https://hal.science/hal-01215580v2}

Submitted on 20 Jan 2016

HAL is a multi-disciplinary open access archive for the deposit and dissemination of scientific research documents, whether they are published or not. The documents may come from teaching and research institutions in France or abroad, or from public or private research centers.
L'archive ouverte pluridisciplinaire HAL, est destinée au dépôt et à la diffusion de documents scientifiques de niveau recherche, publiés ou non, émanant des établissements d'enseignement et de recherche français ou étrangers, des laboratoires publics ou privés. 


\title{
A comparison between two-scale asymptotic expansions and Bloch wave expansions for the homogenization of periodic structures
}

\author{
G. Allaire* M. Briane ${ }^{\dagger} \quad$ M. Vanninathan ${ }^{\ddagger}$ \\ January 13, 2016
}

\begin{abstract}
In this paper we make a comparison between the two-scale asymptotic expansion method for periodic homogenization and the so-called Bloch wave method. It is wellknown that the homogenized tensor coincides with the Hessian matrix of the first Bloch eigenvalue when the Bloch parameter vanishes. In the context of the two-scale asymptotic expansion method, there is the notion of high order homogenized equation [6] where the homogenized equation can be improved by adding small additional higher order differential terms. The next non-zero high order term is a fourth-order term, accounting for dispersion effects (see e.g. [24], [19], [16]). Surprisingly, this homogenized fourth-order tensor is not equal to the fourth-order tensor arising in the Taylor expansion of the first Bloch eigenvalue, which is often called Burnett tensor. Here, we establish an exact relation between the homogenized fourth-order tensor and the Burnett fourth-order tensor. It was proved in [12] that the Burnett fourth-order tensor has a sign. For the special case of a simple laminate we prove that the homogenized fourth-order tensor may change sign. In the elliptic case we explain the difference between the homogenized and Burnett fourthorder tensors by a difference in the source term which features an additional corrector term. Finally, for the wave equation, the two fourth-order tensors coincide again, so dispersion is unambiguously defined, and only the source terms differ as in the elliptic case.
\end{abstract}

Keywords: periodic structure, homogenization, asymptotic expansion, Bloch waves, Burnett coefficients, dispersion

Mathematics Subject Classification: 35B27, 49K20

\section{Introduction}

In the framework of periodic homogenization, the aim of this paper is to compare the method of two-scale asymptotic expansions [6], [7], [23] and that of Bloch wave decomposition [7], [10],

*CMAP, École Polytechnique, CNRS UMR 7641, Université Paris-Saclay, 91128 Palaiseau, FRANCE gregoire.allaire@polytechnique.fr

${ }^{\dagger}$ INSA de Rennes, Institut de Recherche Mathématique de Rennes, FRANCE - mbriane@insa-rennes.fr,

‡TIFR-CAM, Bangalore, INDIA - vanni@math.tifrbng.res.in 
[22], [27]. More precisely, we focus on the modelling of so-called dispersive effects, based on fourth-order homogenized operators. The goal of periodic homogenization is to find the limit of the sequence of solutions $u_{\varepsilon}$ of the conductivity equation with $\varepsilon Y$-periodic coefficients

$$
-\operatorname{div}\left(a_{\varepsilon} \nabla u_{\varepsilon}\right)=f, \quad \text { where } \quad a_{\varepsilon}(x):=a\left(\frac{x}{\varepsilon}\right),
$$

with $a(y)$ a $Y$-periodic symmetric tensor and $Y$ the unit torus $\mathbb{R}^{d} / \mathbb{Z}^{d}$ identified with $[0,1]^{d}$. Equation (1.1) takes place in an open set $\Omega \subset \mathbb{R}^{d}$, which may be bounded or not. Although the method can handle any domain $\Omega$, to avoid any issues with the boundary conditions for (1.1), we assume that either $\Omega=\mathbb{R}^{d}$ or $\Omega$ is a cube with periodic boundary conditions. In the latter case the solution of (1.1) is forced to have zero average in $\Omega$ in order to ensure uniqueness of the solution. Our presentation avoids, as much as possible, any mathematical technical difficulties and stays at a formal level. The precise choice of function spaces, the convergence proofs and error estimates are anyway classical and our main results are not in this framework. Rather we focus on formulas for (high order) homogenized tensors and their interpretation for the modelling of dispersive effects for wave propagation in periodic media. Roughly speaking, dispersion is the phenomenon by which waves with different wavelengths propagate with different velocities. In a linear regime it can occur only in heterogeneous media.

In Section 2 we revisit the two-scale asymptotic expansion method, following the work and notations of [7] (see also [6], [23]). We recall the definition of various homogenized tensors which play a role in the concept of "high order homogenized equation" (see Remark 2.6). In particular, the usual homogenized equation,

$$
-\operatorname{div}\left(a^{*} \nabla u\right)=f
$$

can be improved by adding a small fourth-order operator,

$$
-\operatorname{div}\left(a^{*} \nabla v_{\varepsilon}\right)-\varepsilon^{2} \mathbb{B}^{*} \nabla^{4} v_{\varepsilon}=f
$$

in the sense that $v_{\varepsilon}$ is a "better" approximation of $u_{\varepsilon}$ than $u$. The fourth-order tensor $\mathbb{B}^{*}$ is defined explicitly by a formula involving higher order cell correctors. It is interpreted as modelling dispersive effects (see e.g. [24], [17], [19], [16], [2]).

In Section 3 we review the classical theory of Bloch waves [7], [10], [22], [27] and the Taylor expansion of the first Bloch eigenvalue obtained in [12] which also defines another sequence of tensors. As is well-known, the second-order tensors of both methods agree (namely, the homogenized matrix is equal to the Hessian of the first Bloch eigenvalue), and the third-order tensors are both equal to 0. However, in Section 4 we prove that the fourth-order tensors are in general different (see Theorem 4.1). The fourth-order Burnett tensor $\mathbb{D}^{*}$ of the Bloch expansion is non-positive [12] while the fourth-order homogenized dispersive tensor $\mathbb{B}^{*}$ (obtained by the two-scale asymptotic expansion method) has no fixed sign. In fact, the tensor $\mathbb{B}^{*}$ takes both positive and negative values on layered structures (see Lemma 4.4). As a byproduct we prove in Lemma 4.3 that $\mathbb{B}^{*}$ vanishes in one space dimension. Even more, we indicate in Remark 4.5 that, in the 1-d case, all homogenized tensors of order $\geq 3$ of the two-scale asymptotic expansion (which are actually scalars) are equal to 0 . This is not the case with the tensors of the Bloch expansion in 1-d (see [13], [14], [15]).

In Section 5 we give an explanation of the differences between the two methods. As already said, the fourth-order tensors $\mathbb{B}^{*}$ and $\mathbb{D}^{*}$ give rise to so-called "high order homogenized equations" which are better approximations than the usual homogenized equation. In Proposition 5.2 we prove that these two "high order homogenized equations" coincide if the source term $f(x)$ is replaced by its corrected version $f(x)-\varepsilon \chi^{(1)}\left(\frac{x}{\varepsilon}\right) \cdot \nabla f(x)$ in the two-scale asymptotic 
case. Here $\chi^{1}$ is the usual cell corrector. The "high order homogenized equation" is here a fourth-order partial differential equation which is not well-posed in general. We explain in Remark 5.3 how to modify it to make it well-posed by using a Boussinesq trick as in [9], [17], [19], [16], [1], [2] (the same type of ideas appears also in the study of continuum limits of discrete spring-mass lattices [20]). Eventually Section 6 investigates wave propagation in periodic media, a setting in which dispersive effects are well explained by the "high order homogenized equation" [24], [17], [20], [2]. The situation is simpler than in the elliptic case, in the sense that we prove that the two fourth-order tensors coincide here and are given by the same formula than that of $\mathbb{D}^{*}$ in the elliptic case. In such a case, the fourth-order homogenized tensor, being unambiguously defined, merits its name and interpretation as dispersive tensors. More precisely, in the absence of source term, namely for a system driven only by its initial conditions, the two high order homogenized equations coincide. However, if a source term is present, then it must be corrected as in the elliptic case for the method of two-scale asymptotic expansion to coincide with the Bloch wave method. As a conclusion the Burnett tensor $\mathbb{D}^{*}$ is in all cases the correct fourth-order tensor to model dispersion effects. After this work was completed we learned that similar results have independently been obtained in [3].

\section{Notations}

- $\left(e_{1}, \ldots, e_{d}\right)$ denotes the canonical basis of $\mathbb{R}^{d}$.

- $Y=[0,1]^{d}$ denotes the unit cube of $\mathbb{R}^{d}$.

- $H_{\sharp}^{1}(Y)$ denotes the space of $Y$-periodic functions in $H_{\mathrm{loc}}^{1}\left(\mathbb{R}^{d}\right)$, and $H_{\sharp, 0}^{1}(Y)$ denotes the subspace of $H_{\sharp}^{1}(Y)$ composed of functions with zero $Y$-average.

- For any $n$-order tensor $C=\left[C_{i_{1} \cdots i_{n}}\right]_{1 \leq i_{1}, \ldots, i_{n} \leq d}$, the symmetrization of $C$ is denoted by

$$
C^{S}=\left(\frac{1}{n !} \sum_{\sigma \in \mathfrak{S}_{n}} C_{\sigma\left(i_{1}\right) \cdots \sigma\left(i_{n}\right)}\right)_{1 \leq i_{1}, \ldots, i_{n} \leq d},
$$

where $\mathfrak{S}_{n}$ is the permutations group of order $n$. In the sequel it is understood that all tensors are symmetrized: more precisely, a tensor $C$ is systematically identified with its symmetrized counterpart $C^{S}$.

- In the sequel the summation convention with respect to repeated indices is used.

\section{A review of two-scale asymptotic expansions}

Let $a$ be a $Y$-periodic symmetric matrix-valued such that, for $0<\alpha \leq \beta$,

$$
\alpha I_{d} \leq a(y) \leq \beta I_{d} \quad \text { for a.e. } y \in Y
$$

Consider the oscillating operator $A_{\varepsilon}:=-\operatorname{div}\left(a\left(\varepsilon^{-1} x\right) \nabla \cdot\right)$. When applied to functions of the type $u\left(x, \varepsilon^{-1} x\right)$, where $y \rightarrow u(x, y)$ is a $Y$-periodic function, it can be identified to $A_{\varepsilon}=$ $\varepsilon^{-2} A_{y y}+\varepsilon^{-1} A_{x y}+A_{x x}$ with

$$
\left\{\begin{aligned}
A_{y y} & :=-\operatorname{div}_{y}\left(a(y) \nabla_{y} \cdot\right), \\
A_{x y} & :=-\operatorname{div}_{x}\left(a(y) \nabla_{y} \cdot\right)-\operatorname{div}_{y}\left(a(y) \nabla_{x} \cdot\right), \\
A_{x x} & :=-\operatorname{div}_{x}\left(a(y) \nabla_{x} \cdot\right) .
\end{aligned}\right.
$$


Following [7], the solution $u_{\varepsilon}$ of $A_{\varepsilon} u_{\varepsilon}=f$ in a domain with appropriate boundary condition can be heuristically computed as the sum of the following formal series:

$$
u_{\varepsilon}(x)=\sum_{n=0}^{\infty} \varepsilon^{n} u_{n}\left(x, \frac{x}{\varepsilon}\right),
$$

where $u_{n}(x, \cdot)$ are $Y$-periodic and (ordering the powers of $\varepsilon$ ) satisfy the cascade of equations

$$
\begin{cases}\operatorname{order} \varepsilon^{-2}, & 0=A_{y y} u_{0} \\ \operatorname{order} \varepsilon^{-1}, & 0=A_{y y} u_{1}+A_{x y} u_{0} \\ \operatorname{order} \varepsilon^{0}, & f=A_{y y} u_{2}+A_{x y} u_{1}+A_{x x} u_{0} \\ \operatorname{order} \varepsilon^{1}, & 0=A_{y y} u_{3}+A_{x y} u_{2}+A_{x x} u_{1} \\ & \vdots \\ \operatorname{order} \varepsilon^{n}, & 0=A_{y y} u_{n+2}+A_{x y} u_{n+1}+A_{x x} u_{n}, \quad \text { for } n \geq 1 \\ & \vdots\end{cases}
$$

The ansatz (2.2) is formal since, not only the series does not converge, but it lacks additional boundary layer terms in case of a bounded domain. Furthermore, it requires infinite smoothness of the homogenized solution and thus of the source term $f(x)$. In any case, we refer the reader to [6], [7], [4], [26] for the mathematical justification of this formal process. Again following [7] we compute successively the functions $u_{n}$ introducing suitable cell functions and $n$-order tensors. To this end we need the following lemma, called Fredholm alternative (see [6], [7], [23] for a proof).

Lemma 2.1. For $g(y) \in L^{2}(Y)$, consider the following problem

$$
\left\{\begin{aligned}
&-\operatorname{div}_{y}\left(a(y) \nabla_{y} w\right)=g \text { in } Y, \\
& y \mapsto w(y) \quad Y \text {-periodic. }
\end{aligned}\right.
$$

It admits a unique solution $w(y) \in H_{\sharp, 0}^{1}(Y)$ if and only if the right hand side satisfies the following compatibility condition

$$
\int_{Y} g(y) d y=0
$$

Thanks to Lemma 2.1 we now deduce from (2.3) the formulas for successive terms in the series (2.2).

Computation of $u_{0}$ : it is clear that

$$
u_{0}(x, y)=u(x)
$$

Computation of $u_{1}$ : by linearity we obtain

$$
u_{1}(x, y)=-\chi_{i}(y) \frac{\partial u}{\partial x_{i}}(x)+\tilde{u}_{1}(x)
$$

where $\chi_{i}$ and $\chi_{\eta}^{(1)}=\sum_{i=1}^{d} \eta_{i} \chi_{i}$ are solutions in $H_{\sharp, 0}^{1}(Y)$ of the equations

$$
A_{y y} \chi_{i}=-\operatorname{div}_{y}\left(a e_{i}\right) \quad \text { and } \quad A_{y y} \chi_{\eta}^{(1)}=-\operatorname{div}_{y}(a \eta), \quad \text { for } \eta \in \mathbb{R}^{d} .
$$


Computation of $u_{2}$ : the third equation of (2.3) has a solution if and only if $f-A_{x y} u_{1}-A_{x x} u_{0}$ has a zero $Y$-average, which leads to

$$
-\operatorname{div}\left(a^{*} \nabla u\right)=f
$$

where the homogenized symmetric matrix $a^{*}$ is given by

$$
a^{*} \eta:=\int_{Y}\left(a \eta-a \nabla \chi_{\eta}^{(1)}\right) d y, \quad \text { for } \eta \in \mathbb{R}^{d} .
$$

Next, by the third equation of (2.3) and (2.5) we have

$$
\begin{aligned}
A_{y y} u_{2} & =f-\operatorname{div}_{x}\left(\frac{\partial u}{\partial x_{i}} a \nabla_{y} \chi_{i}\right)-\operatorname{div}_{y}\left(a \chi_{i} \frac{\partial}{\partial x_{i}}\left(\nabla_{x} u\right)\right)+\operatorname{div}_{y}\left(a \nabla_{x} \tilde{u}_{1}\right)+\operatorname{div}_{x}\left(a \nabla_{x} u\right) \\
& =f+\left[a_{i j}-a \nabla_{y} \chi_{i} \cdot e_{j}-\operatorname{div}_{y}\left(\chi_{i} a e_{j}\right)\right] \frac{\partial^{2} u}{\partial x_{i} x_{j}}+\operatorname{div}_{y}\left(a e_{j}\right) \frac{\partial \tilde{u}_{1}}{\partial x_{j}}
\end{aligned}
$$

Hence, defining

$$
b_{i j}:=a_{i j}-a \nabla \chi_{i} \cdot e_{j}-\operatorname{div}\left(\chi_{i} a e_{j}\right), \quad \text { with } \quad \int_{Y} b_{i j}=a_{i j}^{*}, \quad \text { for } i, j \in\{1, \ldots, d\}
$$

$u_{2}$ can be written

$$
u_{2}(x, y)=\chi_{i j}(y) \frac{\partial^{2} u}{\partial x_{i} x_{j}}(x)-\chi_{i}(y) \frac{\partial \tilde{u}_{1}}{\partial x_{i}}(x)+\tilde{u}_{2}(x)
$$

where the functions $\chi_{i j}$ and $\chi_{\eta}^{(2)}:=\chi_{i j} \eta_{i} \eta_{j}$ are the solutions in $H_{\sharp, 0}^{1}(Y)$ of the equations

$$
A_{y y} \chi_{i j}=b_{i j}-\int_{Y} b_{i j}=b_{i j}-a_{i j}^{*} \quad \text { and } \quad A_{y y} \chi_{\eta}^{(2)}=b \eta \cdot \eta-a^{*} \eta \cdot \eta, \quad \text { for } \eta \in \mathbb{R}^{d} .
$$

Note that only the symmetric part of $b_{i j}$ plays a role in (2.9) and the same is true for $\chi_{i j}$ in (2.11).

Computation of $u_{3}$ : the fourth equation of (2.3) has a solution if and only if $A_{x y} u_{2}+A_{x x} u_{1}$ has a zero $Y$-average, namely by (2.5) and (2.11)

$$
\begin{aligned}
0 & =\int_{Y} \operatorname{div}_{x}\left(a \nabla_{x} u_{1}\right) d y+\int_{Y} \operatorname{div}_{x}\left(a \nabla_{y} u_{2}\right) d y \\
& =\left(\int_{Y} a_{i j} d y\right) \frac{\partial^{2} \tilde{u}_{1}}{\partial x_{i} \partial x_{j}}-\frac{\partial}{\partial x_{i}}\left[\int_{Y} a_{i j} \frac{\partial}{\partial x_{j}}\left(\chi_{k} \frac{\partial u}{\partial x_{k}}\right) d y\right] \\
& +\frac{\partial}{\partial x_{i}}\left(\int_{Y} a_{i j} \frac{\partial \chi_{k \ell}}{\partial y_{j}} \frac{\partial^{2} u}{\partial x_{k} \partial x_{\ell}} d y\right)-\left(\int_{Y} a_{i k} \frac{\partial \chi_{j}}{\partial y_{k}} d y\right) \frac{\partial^{2} \tilde{u}_{1}}{\partial x_{i} \partial x_{j}} d y \\
& =a_{i j}^{*} \frac{\partial^{2} \tilde{u}_{1}}{\partial x_{i} x_{j}}-\left(\int_{Y} a_{i j} \chi_{k} d y\right) \frac{\partial^{3} u}{\partial x_{i} \partial x_{j} \partial x_{k}}+\left(\int_{Y} a_{i \ell} \frac{\partial \chi_{k j}}{\partial y_{\ell}} d y\right) \frac{\partial^{3} u}{\partial x_{i} \partial x_{j} \partial x_{k}}
\end{aligned}
$$

Hence, due to the symmetry of $\nabla^{3} u$ we get that

$$
-\operatorname{div}\left(a^{*} \nabla \tilde{u}_{1}\right)=C^{*} \nabla^{3} u, \quad \text { where } \quad C_{i j k}^{*}:=\int_{Y}\left(a \nabla \chi_{i j} \cdot e_{k}-a_{i j} \chi_{k}\right) d y .
$$


Note again that only the symmetric part of $C^{*}$ plays a role in $(2.13)$. Fix $i, j, k \in\{1, \ldots, d\}$. By (2.6), (2.12) and (2.10) combined with the symmetry of $a$, we have

$$
\begin{aligned}
& \int_{Y} a \nabla \chi_{i j} \cdot e_{k} d y=\int_{Y} a e_{k} \cdot \nabla \chi_{i j} d y=\int_{Y} a \nabla \chi_{k} \cdot \nabla \chi_{i j} d y=\int_{Y} a \nabla \chi_{i j} \cdot \nabla \chi_{k} d y \\
& =\int_{Y} b_{i j} \chi_{k} d y=\int_{Y} a_{i j} \chi_{k} d y-\int_{Y} \chi_{k} a e_{j} \cdot \nabla \chi_{i} d y+\int_{Y} \chi_{i} a e_{j} \cdot \nabla \chi_{k} d y,
\end{aligned}
$$

which yields

$$
C_{i j k}^{*}=\int_{Y} a e_{j} \cdot\left(\chi_{i} \nabla \chi_{k}-\chi_{k} \nabla \chi_{i}\right) d y
$$

Therefore, we obtain that

$$
C_{i j k}^{*}=-C_{k j i}^{*}, \quad \forall i, j, k \in\{1, \ldots, d\},
$$

which implies that $\left[C^{*}\right]^{S}=0$ (this result is already proved in [21]) and, combined with the symmetry of $\nabla^{3} u$ in $(2.13)$, yields

$$
\operatorname{div}\left(a^{*} \nabla \tilde{u}_{1}\right)=0 .
$$

Let us now compute $u_{3}$. By the fourth equation of (2.3), (2.5), (2.11) we have

$$
\begin{aligned}
A_{y y} u_{3} & =\operatorname{div}_{x}\left(a \nabla_{y} u_{2}\right)+\operatorname{div}_{y}\left(a \nabla_{x} u_{2}\right)+\operatorname{div}_{x}\left(a \nabla_{x} u_{1}\right) \\
& =\frac{\partial}{\partial x_{i}}\left(a_{i j} \frac{\partial u_{2}}{\partial y_{j}}\right)+\frac{\partial}{\partial y_{i}}\left(a_{i j} \frac{\partial u_{2}}{\partial x_{j}}\right)+\frac{\partial}{\partial x_{i}}\left(a_{i j} \frac{\partial u_{1}}{\partial x_{j}}\right) \\
& =\frac{\partial}{\partial x_{i}}\left(a_{i j} \frac{\partial \chi_{k \ell}}{\partial y_{j}} \frac{\partial^{2} u}{\partial x_{k} \partial x_{\ell}}\right)-\frac{\partial}{\partial x_{i}}\left(a_{i j} \frac{\partial \chi_{k}}{\partial y_{j}} \frac{\partial \tilde{u}_{1}}{\partial x_{k}}\right) \\
& +\frac{\partial}{\partial y_{i}}\left(a_{i j} \chi_{k \ell} \frac{\partial^{3} u}{\partial x_{j} \partial x_{k} \partial x_{\ell}}\right)-\frac{\partial}{\partial y_{i}}\left(a_{i j} \chi_{k} \frac{\partial^{2} \tilde{u}_{1}}{\partial x_{j} \partial x_{k}}\right)+\frac{\partial a_{i j}}{\partial y_{i}} \frac{\partial \tilde{u}_{2}}{\partial x_{j}} \\
& -\frac{\partial}{\partial x_{i}}\left(a_{i j} \chi_{k} \frac{\partial^{2} u}{\partial x_{j} \partial x_{k}}\right)+\frac{\partial}{\partial x_{i}}\left(a_{i j} \frac{\partial \tilde{u}_{1}}{\partial x_{j}}\right)
\end{aligned}
$$

and

$$
\begin{aligned}
A_{y y} u_{3} & =a_{i j} \frac{\partial \chi_{k \ell}}{\partial y_{j}} \frac{\partial^{3} u}{\partial x_{i} \partial x_{k} \partial x_{\ell}}+\frac{\partial}{\partial y_{i}}\left(a_{i j} \chi_{k \ell}\right) \frac{\partial^{3} u}{\partial x_{j} \partial x_{k} \partial x_{\ell}}-a_{i j} \chi_{k} \frac{\partial^{3} u}{\partial x_{i} \partial x_{j} \partial x_{k}} \\
& +a_{i j} \frac{\partial^{2} \tilde{u}_{1}}{\partial x_{i} \partial x_{j}}-a_{i j} \frac{\partial \chi_{k}}{\partial y_{j}} \frac{\partial^{2} \tilde{u}_{1}}{\partial x_{i} \partial x_{k}}-\frac{\partial}{\partial y_{i}}\left(a_{i j} \chi_{k}\right) \frac{\partial^{2} \tilde{u}_{1}}{\partial x_{j} \partial x_{k}}+\frac{\partial a_{i j}}{\partial y_{i}} \frac{\partial \tilde{u}_{2}}{\partial x_{j}}
\end{aligned}
$$

Hence, defining the functions

$$
c_{i j k}:=a \nabla \chi_{j k} \cdot e_{i}+\operatorname{div}\left(\chi_{j k} a e_{i}\right)-a_{i j} \chi_{k}, \quad \text { for } i, j, k \in\{1, \ldots, d\} .
$$

and using (2.6), (2.10), $u_{3}$ can be written

$$
u_{3}(x, y)=\chi_{i j k}(y) \frac{\partial^{3} u}{\partial x_{i} \partial x_{j} \partial x_{k}}(x)+\chi_{i j}(y) \frac{\partial^{2} \tilde{u}_{1}}{\partial x_{i} \partial x_{j}}(x)-\chi_{i}(y) \frac{\partial \tilde{u}_{2}}{\partial x_{i}}(x)+\tilde{u}_{3}(x),
$$

where the functions $\chi_{i j k}$ are the solutions in $H_{\sharp, 0}^{1}(Y)$ of

$$
A_{y y} \chi_{i j k}=c_{i j k}-\int_{Y} c_{i j k}, \quad \text { for } i, j, k \in\{1, \ldots, d\} .
$$


Again, only the symmetric parts of $c_{i j k}$ and $\chi_{i j k}$ play a role.

Equation of $u_{4}$ : the equation for $n=2$ in (2.3) admits a solution $u_{4}$ if and only if $A_{x y} u_{3}+A_{x x} u_{2}$ has a zero $Y$-average, namely by (2.11) and (2.18)

$$
\begin{aligned}
0 & =\int_{Y} \operatorname{div}_{x}\left(a \nabla_{y} u_{3}\right) d y+\int_{Y} \operatorname{div}_{x}\left(a \nabla_{x} u_{2}\right) d y \\
& =\frac{\partial}{\partial x_{i}}\left(\int_{Y} a_{i j} \frac{\partial u_{3}}{\partial y_{j}} d y\right)+\frac{\partial}{\partial x_{i}}\left(\int_{Y} a_{i j} \frac{\partial u_{2}}{\partial x_{j}} d y\right) \\
& =\left(\int_{Y} a_{i j} \frac{\partial \chi_{k \ell m}}{\partial y_{j}}\right) \frac{\partial^{4} u}{\partial x_{i} \partial x_{k} \partial x_{\ell} \partial x_{m}}+\left(\int_{Y} a_{i j} \chi_{k \ell} d y\right) \frac{\partial^{4} u}{\partial x_{i} \partial x_{j} \partial x_{k} \partial x_{\ell}} \\
& +\left(\int_{Y} a_{i j} \frac{\partial \chi_{k \ell}}{\partial y_{j}} d y\right) \frac{\partial^{3} \tilde{u}_{1}}{\partial x_{i} \partial x_{k} \partial x_{\ell}}-\left(\int_{Y} a_{i j} \chi_{k} d y\right) \frac{\partial^{3} \tilde{u}_{1}}{\partial x_{i} \partial x_{j} \partial x_{k}} \\
& -\left(\int_{Y} a_{i j} \frac{\partial \chi_{k}}{\partial y_{j}} d y\right) \frac{\partial^{2} \tilde{u}_{2}}{\partial x_{i} \partial x_{k}}+\left(\int_{Y} a_{i j} d y\right) \frac{\partial^{2} \tilde{u}_{2}}{\partial x_{i} \partial x_{j}} .
\end{aligned}
$$

Therefore, by (2.8) this can be written

$$
-\operatorname{div}\left(a^{*} \nabla \tilde{u}_{2}\right)=\mathbb{B}^{*} \nabla^{4} u+\left[\int_{Y}\left(a_{i \ell} \frac{\partial \chi_{k j}}{\partial y_{\ell}}-a_{i j} \chi_{k}\right) d y\right] \frac{\partial^{3} \tilde{u}_{1}}{\partial x_{i} \partial x_{j} \partial x_{k}},
$$

where $\mathbb{B}^{*}$ is the fourth-order tensor defined by

$$
\mathbb{B}_{i j k \ell}^{*}:=\int_{Y}\left(a_{i j} \chi_{k \ell}+a_{i m} \frac{\partial \chi_{k \ell j}}{\partial y_{m}}\right) d y, \quad \text { for } i, j, k, \ell \in\{1, \ldots, d\} .
$$

Note again that only the symmetric part of $\mathbb{B}^{*}$ plays a role in $(2.20)$.

The last term in the right hand side of $(2.20)$ is (again by symmetry in $i, j, k)$ equal to $C^{*} \nabla^{3} \tilde{u}_{1}$ which vanishes by virtue of (2.15). Therefore, (2.20) simplifies to

$$
-\operatorname{div}\left(a^{*} \nabla \tilde{u}_{2}\right)=\mathbb{B}^{*} \nabla^{4} u
$$

Remark 2.2. For the sake of simplicity in the notations we did not explicitly symmetrize all tensors which are contracted with (symmetric) derivative tensors $\nabla_{x}^{k}$ acting on some macroscopic functions. Nevertheless, recall that, by construction, any tensor should be identified with its symmetric part. Note also the sign convention for the first-order corrector $\chi_{i}(y)$ which is the opposite of the higher order correctors $\chi_{i j}, \chi_{i j k}$. This is due to our adhering to the notations in [7].

Remark 2.3. Of course, the above process of computing successive terms in the ansatz (2.2) works without any symmetry assumption on the coefficient matrix $a(y)$. Nevertheless we made a symmetry assumption because we need it for the next section on Bloch waves.

Remark 2.4. If we continue the above procedure assuming that $u$ is smooth enough, similarly to $(2.5),(2.11),(2.18)$ we get that for any integer $n \geq 1$, the $n$-order term of the asymptotic expansion (2.2) can be expressed as

$$
u_{n}(x, y)=\chi^{n}(y) \cdot \nabla^{n} u(x)+\sum_{k=1}^{n-1} \chi^{k}(y) \cdot \nabla^{n-k} \tilde{u}_{k}(x)+\tilde{u}_{n}(x), \quad \text { with } \tilde{u}_{k}(x):=\int_{Y} u_{k}(x, y) d y,
$$

and $\chi^{k}$, for $k \in\{1, \ldots, n\}$, are $k$-order tensor-valued functions in $H_{\sharp, 0}^{1}(Y)^{d^{k}}$ and $\cdot$ denotes full contraction of these tensors. For example, we have $\chi^{1}=\left(\chi_{1}, \ldots, \chi_{d}\right), \chi^{2}=\left(\chi_{i j}\right)_{1 \leq i, j \leq d}$ and so 
on. Similarly to $(2.13),(2.20)$ there also exist $k$-order tensors $\mathbb{B}_{k}^{*}$, for $k \in\{3, \ldots, n+2\}$, such that

$$
-\operatorname{div}\left(a^{*} \nabla \tilde{u}_{n}\right)=\mathbb{B}_{n+2}^{*} \nabla^{n+2} u+\sum_{k=1}^{n-1} \mathbb{B}_{n+2-k}^{*} \nabla^{n+2-k} \tilde{u}_{k},
$$

which in particular satisfy $\mathbb{B}_{3}^{*}=C^{*}=0$ by $(2.16)$ and $\mathbb{B}_{4}^{*}=\mathbb{B}^{*}$ by $(2.20)$.

Remark 2.5. If one is interested in an infinite order asymptotic expansion of the solution, the approach of [6] is slightly simpler than that of [7]. Indeed, the authors in [6] prove that the ansatz (2.2) is equivalent to

$$
u_{\varepsilon}(x)=\sum_{n=0}^{\infty} \varepsilon^{n} \chi^{n}\left(\frac{x}{\varepsilon}\right) \cdot \nabla^{n} U_{\varepsilon}(x)
$$

where $\chi^{n}$ is the $n$-order corrector introduced in Remark 2.4 (with the convention that $\chi^{0} \equiv 1$ ) and

$$
U_{\varepsilon}(x)=u(x)+\sum_{n \geq 1} \varepsilon^{n} \tilde{u}_{n}(x),
$$

where each $\tilde{u}_{n}$ is defined by (2.23)-(2.24). Since all correctors functions $\chi^{n}$, for $n \geq 1$, have zero average, the macroscopic (and non oscillating) function $U_{\varepsilon}(x)$ in $(2.25)$ can be interpreted as an amplitude or profile function, which is a better approximation of the exact solution $u_{\varepsilon}$ of (1.1) than just the homogenized solution $u$, solution of (2.7).

Remark 2.6. Writing an effective equation for a truncated version of the non oscillating ansatz $U_{\varepsilon}$, defined by (2.26), has been studied in various settings (see [6], [24], [19], [16], [25]) under the name of "higher order homogenization". Here we give the "second order" homogenized equation which is a proposed explanation of dispersive effects for wave propagation in periodic media [24], [19], [16], [1], [2] or of second gradient theory in mechanics [25]. In other words, we truncate the non oscillating ansatz $(2.26)$ at second order. We also assume that $\Omega=\mathbb{R}^{d}$ or $\Omega$ is a cube with periodic boundary conditions and zero-average solutions. In such a case, equation (2.16) for $\tilde{u}_{1}(x)$ admits 0 as its unique solution. Therefore, (2.26) becomes

$$
U_{\varepsilon}(x)=v_{\varepsilon}(x)+O\left(\varepsilon^{3}\right) \quad \text { with } \quad v_{\varepsilon}(x)=u(x)+\varepsilon^{2} \tilde{u}_{2}(x) .
$$

Then adding the homogenized equation (2.7) with (2.22) multiplied by $\varepsilon^{2}$ yields

$$
-\operatorname{div}\left(a^{*} \nabla v_{\varepsilon}\right)-\varepsilon^{2} \mathbb{B}^{*} \nabla^{4} v_{\varepsilon}=f+O\left(\varepsilon^{4}\right)
$$

Neglecting the term of order $\varepsilon^{4}$ in (2.27) gives the "second order" homogenized equation (1.2), as announced in the introduction. If the tensor $\mathbb{B}^{*}$ were non-positive, equation (2.27) would be well-posed. Unfortunately, as we shall see in Section $4, \mathbb{B}^{*}$ has no sign in full generality and (2.27) is thus not well-posed. We shall see in the sequel (see Remark 5.3) how to modify it to make it well-posed by using a Boussinesq trick [9].

Remark 2.7. It is customary to enforce the normalization condition that all solutions of the cell problems have zero average, as is the case in Lemma 2.1. It is clear that the results of homogenization must not depend on the normalization. However, some of the quantities, defined above, may vary according to the choice of the additive constants in the definition of the cell solutions. We now summarize the main changes in such a case. For some constants $\mathfrak{c}_{i} \in \mathbb{R}$, we replace the solution $\chi_{i}(y)$ of the first-order cell problem $(2.6)$ by $\chi_{i}(y)+\mathfrak{c}_{i}$, which is another possible solution. Clearly, definition (2.8) of the homogenized tensor $a^{*}$ does not depend on the 
choice of $\mathfrak{c}_{i}$. However, in (2.5) the function $\tilde{u}_{1}$ should be replaced by $\tilde{u}_{1}+\mathfrak{c}_{i} \frac{\partial u}{\partial x_{i}}$ and in (2.10) the function $b_{i j}$ should be replaced by $b_{i j}-\mathfrak{c}_{i} \operatorname{div}_{y}\left(a e_{j}\right)$. Thus, for some constants $\mathfrak{c}_{i j} \in \mathbb{R}$, the solution $\chi_{i j}(y)$ of the second order cell problem (2.12) should be replaced by $\chi_{i j}(y)+\mathfrak{c}_{i} \chi_{j}+\mathfrak{c}_{i j}$, which is another possible solution. Then, the tensor $C_{i j k}^{*}$, defined by (2.13), should be replaced by $C_{i j k}^{*}-\mathfrak{c}_{i} a_{j k}^{*}$, which ensures that equation (2.16) for $\tilde{u}_{1}$ is unchanged. In turn, the source term $c_{i j k}$, defined by (2.17), has to be replaced by $c_{i j k}-\mathfrak{c}_{k} b_{i j}+\mathfrak{c}_{j k} \operatorname{div}_{y}\left(a e_{i}\right)$ and, as a consequence, for some constant $\mathfrak{c}_{i j k} \in \mathbb{R}$, the solution $\chi_{i j k}(y)$ of the third-order cell problem (2.19) becomes $\chi_{i j k}(y)-\mathfrak{c}_{k} \chi_{i j}-\mathfrak{c}_{j k} \chi_{i}+\mathfrak{c}_{i j k}$. Eventually, the fourth-order tensor $\mathbb{B}_{i j k \ell}^{*}$, defined by (2.21), is replaced by $\mathbb{B}_{i j k \ell}^{*}+a_{i j}^{*} \mathfrak{c}_{k l}$. Note that equation (2.22) will remain valid with the new tensor $\mathbb{B}_{i j k \ell}^{*}+a_{i j}^{*} \mathfrak{c}_{k l}$ since, accordingly, $\tilde{u}_{2}$ is replaced by $\tilde{u}_{2}+\mathfrak{c}_{i} \frac{\partial \tilde{u}_{1}}{\partial x_{i}}-\mathfrak{c}_{i j} \frac{\partial^{2} u}{\partial x_{i} \partial x_{j}}$.

\section{A review of Bloch wave expansions}

While the two-scale asymptotic expansion method of Section 2 was performed in the physical $x$-space, the Bloch wave method works in the dual or Fourier space. To this end we consider the spectral resolution of the operator $A_{\varepsilon}$. Because of the symmetry of the matrix $a(y)$, the operator $A_{\varepsilon}$ defines an unbounded self-adjoint operator in $L^{2}\left(\mathbb{R}^{d}\right)$. By the change of variables $y=x / \varepsilon$, we are reduced to consider the operator $A_{y y}$. Bloch waves are eigenvectors of $A_{y y}$ satisfying a special boundary condition and we are interested in their energies or eigenvalues.

In order to take into account the impact of the $\mathbb{Z}^{d}$-translation invariance of the coefficients of $A_{y y}$ on its spectrum, the Bloch wave method introduces the following spectral problem. For a given parameter $\eta \in \mathbb{R}^{d}$, find eigenvalues $\lambda=\lambda(\eta)$ in $\mathbb{R}$ and eigenvectors $\psi=\psi(\eta)$ in $H_{\text {loc }}^{1}\left(\mathbb{R}^{d}\right)$ satisfying

$$
A_{y y} \psi=\lambda \psi, \quad \psi(y+p)=e^{2 \pi i p \cdot \eta} \psi(y) \quad \forall y \in \mathbb{R}^{d}, p \in \mathbb{Z}^{d} .
$$

Since the above quasi-periodic condition remains invariant under integer translation in $\eta$, it is sufficient to take $\eta$ in $Y$. Note that, with our notations (featuring a $2 \pi$ factor in the phase), the primary and dual cells coincide. To simplify the boundary conditions we make the following change of unknowns

$$
\psi(y, \eta)=e^{2 \pi i y \cdot \eta} \phi(y, \eta)
$$

Then $\phi$ is $Y$-periodic and satisfies

$$
A(\eta) \phi=\lambda(\eta) \phi \quad \forall y \in Y
$$

where $A(\eta)$ is the translated (or shifted) operator defined by

$$
A(\eta):=-\left(\frac{\partial}{\partial y_{k}}+2 \pi i \eta_{k}\right)\left[a_{k \ell}\left(\frac{\partial}{\partial y_{\ell}}+2 \pi i \eta_{\ell}\right)\right], \quad \text { with } \quad A(0)=A_{y y} .
$$

The above spectral problem for $A(\eta)$ in the unit torus $Y$, the so-called Bloch problem, is classical both in mathematics [27] and in solid state physics [22]. It is easily seen that $A(\eta)$ is an unbounded self-adjoint operator in $L_{\sharp}^{2}(Y)$ with compact resolvent. Its quadratic form is non-negative and so its eigenvalues are non-negative and discrete. We are interested in the first eigenvalue/ground energy $\lambda_{1}(\eta)$ which is the relevant one in the homogenization process. When $\eta=0$, it is easy to see that $\lambda_{1}(0)=0$ is a simple eigenvalue of $A(0)=A_{y y}$ with constants as eigenfunctions. Regular perturbation theory proves then that $\lambda_{1}(\eta)$ is simple and is analytic in a neighborhood of $\eta=0$.

The derivatives of $\lambda_{1}(\eta)$ at order $k$ at $\eta=0$ define $k$-order tensors which can equivalently be expressed in terms of suitable cell test functions. In the sequel we shall compare them, up 
to fourth-order, with the previous tensors introduced by the method of two-scale asymptotic expansion in Section 2. We now recall some results from [11], [12] about the Taylor expansion of $\lambda_{1}(\eta)$ at $\eta=0$.

Theorem 3.1. All odd order derivatives of $\lambda_{1}(\eta)$ vanish at $\eta=0$. Furthermore, $\lambda_{1}(\eta)$ admits the following fourth-order expansion:

$$
\lambda_{1}(\eta)=4 \pi^{2} a^{*} \eta \cdot \eta+(2 \pi)^{4} \mathbb{D}^{*} \cdot(\eta \otimes \eta \otimes \eta \otimes \eta)+O\left(|\eta|^{6}\right),
$$

where $\frac{1}{8 \pi^{2}} \lambda_{1}^{(2)}(0)=a^{*}$ is the homogenized matrix defined by (2.8) and $\mathbb{D}^{*}$ is the symmetric fourth-order tensor $\frac{1}{4 !(2 \pi)^{4}} \lambda_{1}^{(4)}(0)$ (called Burnett tensor in the literature) which is equivalently defined by

$$
\mathbb{D}_{i j k l}^{*}:=-\int_{Y}\left(a_{i j} \chi_{k \ell}+a_{i m} \frac{\partial \hat{\chi}_{k \ell j}}{\partial y_{m}}\right) d y, \quad \text { for } i, j, k, \ell \in\{1, \ldots, d\} .
$$

In (3.3) the functions $\chi_{i j}$ are defined by (2.12) and $\hat{\chi}_{i j k}$ are the solutions in $H_{\sharp, 0}^{1}(Y)$ of

$$
A_{y y} \hat{\chi}_{i j k}=a_{i j}^{*} \chi_{k}+c_{i j k}-\int_{Y} c_{i j k} d y, \quad \text { for } i, j, k \in\{1, \ldots, d\},
$$

where $\chi_{k}$ are given by (2.6) and $c_{i j k}$ are given by (2.17).

As usual, the tensor $\mathbb{D}^{*}$ and the functions $\hat{\chi}_{i j k}$ are understood as symmetrized. Note that the functions $\hat{\chi}_{i j k}$ are different from the previous ones $\chi_{i j k}$ defined by (2.19) (see (4.3) below).

Remark 3.2. As a by-product of Theorem 3.1 it was shown in [11] that the $\eta$-derivatives of the first eigenfunction $\phi_{1}(y, \eta)$ coincide, up to additive constants, with the solutions of some cell problems. A preliminary step is to choose a proper normalization of the eigenfunction $\phi_{1}(y, \eta)$ so that, first, it is analytic for $\eta$ near 0 and, second, its derivatives will be either real or purely imaginary. Following [11] we choose

$$
\left\|\phi_{1}(y, \eta)\right\|_{L^{2}(Y)}=1 \quad \text { and } \quad \int_{Y} \phi_{1}(y, \eta) d y \in \mathbb{R} .
$$

In particular, it implies that $\phi_{1}(y, 0)=1$. The first condition in (3.5) is classical while the second one is easily obtained as follows. Replace any analytic branch $\eta \rightarrow \phi_{1}(y, \eta)$ by $\phi_{1}(y, \eta) e^{i P(\eta)}$ where $P$ is an analytic function from $\mathbb{R}^{d}$ into $\mathbb{R}$, chosen so that the second condition is satisfied for the product $\phi_{1}(y, \eta) e^{i P(\eta)}$ (this amounts to a simple constant change of phase for the first eigenfunction). Under this normalization, [11] proved that the $\eta$-derivatives of the first eigenfunction $\phi_{1}(y, \eta)$ coincide with the cell solutions

$$
\begin{aligned}
\frac{\partial \phi_{1}}{\partial \eta_{k}}(y, 0) & =-2 \pi i \chi_{k}(y), \quad \frac{\partial^{2} \phi_{1}}{\partial \eta_{k} \partial \eta_{l}}(y, 0)=-8 \pi^{2} \chi_{k l}(y)-4 \pi^{2} \int_{Y} \chi_{k}(y) \chi_{l}(y) d y \\
\frac{\partial^{3} \phi_{1}}{\partial \eta_{j} \partial \eta_{k} \partial \eta_{l}}(y, 0) & =-48 \pi^{3} i\left(\hat{\chi}_{j k l}(y)-\frac{1}{3} \chi_{j}(y) \int_{Y} \chi_{k} \chi_{l} d y-\frac{1}{3} \chi_{k}(y) \int_{Y} \chi_{j} \chi_{l} d y-\frac{1}{3} \chi_{l}(y) \int_{Y} \chi_{k} \chi_{j} d y\right),
\end{aligned}
$$

where $\chi_{k}$ are defined by $(2.6), \chi_{k l}$ by $(2.12)$ and $\hat{\chi}_{j k l}$ by (3.4).

Let us explain in more details the Bloch wave method for the homogenization of the elliptic equation (1.1) in the entire space $\mathbb{R}^{d}$ (it can be extended to the case of the elasticity system as in [18]). First we have the following Bloch wave decomposition result written in rescaled variables $x=\varepsilon y$ and $\xi=\eta / \varepsilon$. 
Lemma 3.3. Any function $f \in L^{2}\left(\mathbb{R}^{d}\right)$ can be decomposed as

$$
f(x)=\sum_{n \geq 1} \int_{\varepsilon^{-1} Y} \alpha_{n}^{\varepsilon}(\xi) \phi_{n}\left(\frac{x}{\varepsilon}, \varepsilon \xi\right) e^{2 \pi i x \cdot \xi} d \xi
$$

where

$$
\alpha_{n}^{\varepsilon}(\xi)=\int_{\mathbb{R}^{d}} f(x) e^{-2 \pi i x \cdot \xi} \bar{\phi}_{n}\left(\frac{x}{\varepsilon}, \varepsilon \xi\right) d x,
$$

and $\phi_{n}(y, \eta)$ is the $n$-th normalized eigenfunction of (3.1). Furthermore, it satisfies Parseval equality

$$
\int_{\mathbb{R}^{d}}|f(x)|^{2} d x=\sum_{n \geq 1} \int_{\varepsilon^{-1} Y}\left|\alpha_{n}^{\varepsilon}(\xi)\right|^{2} d \xi .
$$

Similarly, we decompose the solution $u_{\varepsilon}$ of (1.1) as

$$
u_{\varepsilon}(x)=\sum_{n \geq 1} \int_{\varepsilon^{-1} Y} \hat{u}_{n}^{\varepsilon}(\xi) \phi_{n}\left(\frac{x}{\varepsilon}, \varepsilon \xi\right) e^{2 \pi i x \cdot \xi} d \xi .
$$

Since the eigenbasis $\left\{\phi_{n}\right\}$ diagonalizes the elliptic operator, equation (1.1) reduces to the following algebraic equalities

$$
\varepsilon^{-2} \lambda_{n}(\varepsilon \xi) \hat{u}_{n}^{\varepsilon}(\xi)=\alpha_{n}^{\varepsilon}(\xi)
$$

With the help of energy estimates it was proved in [10] that all modes $n \geq 2$ are negligible and that only the first one $n=1$ matters. Furthermore, as $\varepsilon$ goes to zero, $\alpha_{1}^{\varepsilon}(\xi)$ converges in $L^{2}\left(\mathbb{R}^{d}\right)$ to a limit $\alpha_{1}(\xi)=\hat{f}(\xi)$ which is the Fourier transform of $f(x)$ (see[10] for details).

Thanks to the Taylor expansion (3.2) one deduces that $\hat{u}_{1}^{\varepsilon}(\xi)$ converges in $L_{l o c}^{2}\left(\mathbb{R}^{d}\right)$ to $\hat{u}_{1}(\xi)$ which is given by

$$
a^{*} \xi \cdot \xi \hat{u}_{1}(\xi)=\alpha_{1}(\xi),
$$

which is precisely the Fourier transform of the homogenized equation (2.7). In particular, it implies that $\hat{u}_{1}(\xi)=\hat{u}(\xi)$ is the Fourier transform of the homogenized solution $u(x)$, satisfying the homogenized equation (2.7).

A fundamental property of the Burnett tensor $\mathbb{D}^{*}$, discovered by $[12]$, is that it is nonpositive.

Proposition 3.4 ([12]). The fourth-order tensor $\mathbb{D}^{*}$, defined by (3.3), satisfies for any $\eta \in \mathbb{R}^{d}$

$$
\mathbb{D}^{*}(\eta \otimes \eta):(\eta \otimes \eta)=-\int_{Y} a \nabla\left(\chi_{\eta}^{(2)}-\frac{1}{2}\left(\chi_{\eta}^{(1)}\right)^{2}\right) \cdot \nabla\left(\chi_{\eta}^{(2)}-\frac{1}{2}\left(\chi_{\eta}^{(1)}\right)^{2}\right) d y \leq 0
$$

where $\chi_{\eta}^{(1)}$ is defined by (2.6) and $\chi_{\eta}^{(2)}$ by (2.12).

\section{A comparison of the fourth-order tensors $\mathbb{B}^{*}$ and $\mathbb{D}^{*}$}

The main result of this section is that the two fourth-order tensors $\mathbb{B}^{*}$ and $\mathbb{D}^{*}$ are different.

Theorem 4.1. The fourth-order tensor $\mathbb{B}^{*}$ (2.21) arising in the two-scale asymptotic expansion admits the following decomposition:

$$
\mathbb{B}^{*}=-\mathbb{D}^{*}-\mathbb{E}^{*}, \quad \text { with } \quad \mathbb{E}_{i j k l}^{*}:=a_{k \ell}^{*} d_{i j}^{*} \quad \text { and } \quad d_{i j}^{*}:=\int_{Y} \chi_{i} \chi_{j} d y
$$


and $\mathbb{D}^{*}$ is the Burnett tensor defined by (3.3). Furthermore,

$$
\left\{\begin{array}{l}
\mathbb{D}^{*}(\eta \otimes \eta):(\eta \otimes \eta) \leq 0 \\
\mathbb{E}^{*}(\eta \otimes \eta):(\eta \otimes \eta) \geq 0,
\end{array} \quad \forall \eta \in \mathbb{R}^{d},\right.
$$

while there are examples of tensors $\mathbb{B}^{*}$ which have no fixed sign.

Proof. We compare the tensors $\mathbb{B}^{*}(2.21)$ and $\mathbb{D}^{*}(3.3)$ (all tensors are symmetrized). First, comparing (2.19) and (3.4) we get that

$$
\hat{\chi}_{i j k}=\chi_{i j k}+a_{i j}^{*} w_{k},
$$

where the functions $w_{k}$ are the solutions in $H_{\sharp, 0}^{1}(Y)$ of

$$
A_{y y} w_{k}=\chi_{k} .
$$

Putting (4.3) in (3.3) and comparing to (2.21) it follows that

$$
\mathbb{D}_{i j k \ell}^{*}=-\mathbb{B}_{i j k \ell}^{*}-a_{k \ell}^{*} \int_{Y} a \nabla w_{j} \cdot e_{i} d y
$$

where the last integral becomes, using successively (2.6) and (4.4),

$$
\int_{Y} a \nabla w_{j} \cdot e_{i} d y=\int_{Y} a \nabla w_{j} \cdot \nabla \chi_{i} d y=\int_{Y} \chi_{i} \chi_{j} d y
$$

Therefore, (4.5) is equivalent to the relation $\mathbb{B}^{*}=-\mathbb{D}^{*}-\mathbb{E}^{*}$, which implies that

$$
\mathbb{B}^{*}(\eta \otimes \eta):(\eta \otimes \eta)=-\mathbb{D}^{*}(\eta \otimes \eta):(\eta \otimes \eta)-\left(a^{*} \eta \cdot \eta\right)\left(d^{*} \eta \cdot \eta\right), \quad \forall \eta \in \mathbb{R}^{d},
$$

with

$$
d^{*} \eta \cdot \eta=\int_{Y}\left(\chi_{\eta}^{(1)}\right)^{2} d y
$$

where $\chi_{\eta}^{(1)}$ is defined by (2.6). This implies that $d^{*}$ is non-negative and so is $\mathbb{E}^{*}$. Furthermore, $\mathbb{D}^{*}$ is non-positive in view of Proposition 3.4. The fact that $\mathbb{B}^{*}$ has no sign is investigated in Lemma 4.4 below.

Corollary 4.2. The fourth-order homogenized tensor $\mathbb{B}^{*}$ (2.21) satisfies the following upper and lower bounds

$$
-\mathbb{E}^{*} \leq \mathbb{B}^{*} \leq-\mathbb{D}^{*}
$$

Proof. By virtue of (3.11), (4.7) implies $\mathbb{B}^{*} \geq-\mathbb{E}^{*}$. Moreover, since $\mathbb{E}^{*}$ is nonnegative, we immediately obtain the upper bound in (4.8).

We now apply the above analysis to the case of isotropic layers depending on one direction, say $y_{1}$, namely the conductivity matrix is reduced to $a\left(y_{1}\right) I_{d}$ where $a$ is 1 -periodic on $\mathbb{R}$. In such a case, all cell solutions depends only on the single variable $y_{1}$ and derivatives are denoted by '. In this particular setting we prove that, for a two-phase simple laminate, the fourth-order tensor $\mathbb{B}^{*}$ has no sign. 
Lemma 4.3. For a laminate microstructure, defined by its conductivity tensor $a\left(y_{1}\right) I_{d}$, where $a$ is 1-periodic, the fourth-order homogenized tensor $\mathbb{B}^{*}$ satisfies

$$
\mathbb{B}^{*}(\eta \otimes \eta):(\eta \otimes \eta)=\left|\eta^{\prime}\right|^{4} \int_{0}^{1} a\left(\chi_{22}^{\prime}\right)^{2} d y_{1}+\eta_{1}^{2}\left|\eta^{\prime}\right|^{2} \int_{0}^{1} \chi_{1}\left(2 \underline{a} \chi_{22}^{\prime}-\bar{a} \chi_{1}\right) d y_{1},
$$

for any $\eta=\left(\eta_{1}, \eta^{\prime}\right) \in \mathbb{R}^{d}$.

In particular, in one space dimension, we have $\mathbb{B}^{*}=0$.

Proof. Let $\underline{a}$ be the harmonic mean of $a$, let $\bar{a}$ be the arithmetic mean of $a$, and let $A$ be the 1-periodic function defined by

$$
A\left(y_{1}\right):=\int_{0}^{y_{1}}(a-\bar{a}) d t, \quad \text { for } y_{1} \in \mathbb{R}
$$

A simple computation yields for the functions (2.6) and (2.12),

$$
\left\{\begin{array} { l } 
{ \chi _ { 1 } ^ { \prime } ( y _ { 1 } ) = - \frac { \underline { a } } { a ( y _ { 1 } ) } + 1 } \\
{ \chi _ { i } ( y _ { 1 } ) = 0 \text { if } i > 1 }
\end{array} \text { and } \left\{\begin{array}{l}
\chi_{11}^{\prime}\left(y_{1}\right)=\chi_{1}\left(y_{1}\right) \\
\chi_{i i}^{\prime}\left(y_{1}\right)=-\frac{A\left(y_{1}\right)}{a\left(y_{1}\right)}+\frac{\underline{a}}{a\left(y_{1}\right)} \int_{0}^{1} \frac{A}{a} d y_{1} \quad \text { if } i>1 \\
\chi_{i j}\left(y_{1}\right)=0 \text { if } i \neq j,
\end{array}\right.\right.
$$

and the homogenized matrix is given by

$$
a^{*}=\underline{a}\left(e_{1} \otimes e_{1}\right)+\bar{a} \sum_{i=2}^{d}\left(e_{i} \otimes e_{i}\right) .
$$

Putting this in (4.1) and (3.11) we have for any $\eta=\left(\eta_{1}, \eta^{\prime}\right) \in \mathbb{R}^{d}$,

$$
\begin{aligned}
\mathbb{B}^{*}(\eta \otimes \eta):(\eta \otimes \eta) & =\int_{0}^{1} a\left(\eta_{1}^{2} \chi_{11}^{\prime}+\left|\eta^{\prime}\right|^{2} \chi_{22}^{\prime}-\eta_{1}^{2} \chi_{1} \chi_{1}^{\prime}\right)^{2} d y_{1}-\eta_{1}^{2}\left(\underline{a} \eta_{1}^{2}+\bar{a}\left|\eta^{\prime}\right|^{2}\right) \int_{0}^{1}\left(\chi_{1}\right)^{2} d y_{1} \\
& =\eta_{1}^{4} \int_{0}^{1} \chi_{1}^{2}\left[a\left(1-\chi_{1}^{\prime}\right)^{2}-\underline{a}\right] d y_{1}+\left|\eta^{\prime}\right|^{4} \int_{0}^{1} a\left(\chi_{22}^{\prime}\right)^{2} d y_{1} \\
& +\eta_{1}^{2}\left|\eta^{\prime}\right|^{2} \int_{0}^{1} \chi_{1}\left[2 a\left(1-\chi_{1}^{\prime}\right) \chi_{22}^{\prime}-\bar{a} \chi_{1}\right] d y_{1} .
\end{aligned}
$$

Since by the periodicity of $\chi_{1}$

$$
\int_{0}^{1} \chi_{1}^{2}\left[a\left(1-\chi_{1}^{\prime}\right)^{2}-\underline{a}\right] d y_{1}=-\underline{a} \int_{0}^{1} \chi_{1}^{\prime} \chi_{1}^{2} d y_{1}=0,
$$

we finally obtain (4.9).

Now, consider the case of a two-phase material with phases $\alpha<\beta$ and volume fraction $\theta$, which is studied in [15]. Namely, from now on, we assume, for $0<\theta<1$,

$$
a(y)= \begin{cases}\alpha & \text { if } 0<y_{1}<\theta \\ \beta & \text { if } \theta<y_{1}<1\end{cases}
$$

Lemma 4.4. Assume $d \geq 2$. For a simple two-phase laminate defined by (4.12) the quartic form $\eta \rightarrow \mathbb{B}^{*}(\eta \otimes \eta):(\eta \otimes \eta)$ takes positive and negative values. 
Proof. In this case, some algebra leads to

$$
\chi_{1}=\frac{\underline{a}}{\alpha \beta}(A-\bar{A}) \quad \text { and } \quad \chi_{22}^{\prime}=\frac{-1}{a}(A-\bar{A}), \quad \text { with } \bar{A}:=\int_{0}^{1} A,
$$

and

$$
\int_{0}^{1} \frac{A}{a}=\frac{\bar{A}}{\underline{a}}, \quad \int_{0}^{1} \frac{(A-\bar{A})^{2}}{a}=\frac{1}{\underline{a}} \int_{0}^{1}(A-\bar{A})^{2}, \quad \int_{0}^{1}(A-\bar{A})^{2}=\frac{1}{12} \theta^{2}(1-\theta)^{2}(\beta-\alpha)^{2} .
$$

Then, the previous formula (4.9) is reduced to

$$
\mathbb{B}^{*}(\eta \otimes \eta):(\eta \otimes \eta)=\left|\eta^{\prime}\right|^{2}\left(\frac{1}{\underline{a}}\left|\eta^{\prime}\right|^{2}-\frac{\underline{a}^{2}}{(\alpha \beta)^{2}}[(2-\theta) \alpha+(1+\theta) \beta] \eta_{1}^{2}\right) \int_{0}^{1}(A-\bar{A})^{2} .
$$

First, choosing $\eta_{1}=0,\left|\eta^{\prime}\right|=1$, (4.14) yields $\mathbb{B}^{*}(\eta \otimes \eta):(\eta \otimes \eta)>0$. Next, choosing $\left|\eta^{\prime}\right|=1$ and $\eta_{1}$ large enough, (4.14) gives $\mathbb{B}^{*}(\eta \otimes \eta):(\eta \otimes \eta)<0$. Therefore, contrary to $\mathbb{D}^{*}$ the tensor $\mathbb{B}^{*}$ does not have a constant sign with respect to the direction $\eta$.

Remark 4.5. Lemma 4.3 shows that $\mathbb{B}^{*}=0$ in 1 -d. Actually much more is true: in 1 -d, all higher order homogenized coefficients $\mathbb{B}_{n}^{*}$ (defined in Remark 2.4) vanish. Indeed, by simple computations one can show that the correctors or cell solutions $\chi^{n} \in H_{\sharp, 0}^{1}([0,1])$, for $n \geq 1$, can be obtained by the following iterative procedure:

$$
\left\{\begin{array}{l}
\chi^{\prime 1}(y)=1-\frac{\underline{a}}{a(y)} \\
\chi^{\prime 2}(y)=\chi^{1}(y) \\
\chi^{\prime n+1}(y)=-\chi^{n}(y) \text { if } n \geq 2 .
\end{array}\right.
$$

Furthermore, if the solution $u$ of the homogenized equation,

$$
-\underline{a} u^{\prime \prime}(x)=f(x),
$$

is smooth, $u \in C^{\infty}(\mathbb{R})$, then, for any $n \geq 1$, the solutions of the cascade of equations (2.3) are given by

$$
\left\{\begin{array}{l}
u_{0}(x, y)=u(x) \\
u_{1}(x, y)=-\chi^{1}(y) u^{\prime}(x) \\
u_{n}(x, y)=\chi^{n}(y) u^{(n)}(x) \text { if } n \geq 2 .
\end{array}\right.
$$

Comparing (4.16) with (2.23), we deduce that the functions $\tilde{u}_{k}$ and the tensors $\mathbb{B}_{n}^{*}$ of $(2.24)$ are equal to 0 in dimension one.

Conversely, if we consider any family of functions $u_{n}$ satisfying (2.3), then following the procedure of Section 2 we get that for any $n \geq 1$, the function $\tilde{u}_{n}$ of $(2.23)$ solves the equation

$$
-\underline{a} \tilde{u}_{n}^{\prime \prime}(x)=0
$$

and thus are affine. Hence, from this we deduce that the general expansion (2.23) is reduced to

$$
u_{n}(x, y)=\hat{u}_{n}(x, y)-\alpha_{n-1} \chi_{1}(y)+\alpha_{n} x+\beta_{n}, \quad \forall n \geq 2,
$$

where $\alpha_{n}, \beta_{n}$ are constants. 


\section{An explanation of the differences}

In this section we provide an explanation for the differences obtained in the fourth-order tensors $\mathbb{D}^{*}$ and $\mathbb{B}^{*}$. The key idea is that the two-scale asymptotic expansion method and the Bloch wave method does not treat exactly the same right hand sides. This (small) difference between the source terms is at the root of the different fourth-order dispersion tensors. We explain it in a formal way for the sake of simplicity (see Remark 5.4 at the end of this section for references about rigorous proofs).

In the framework of the Bloch wave method, instead of equation (1.1) with a right hand side $f$, independent of $\varepsilon$, consider rather the following equation

$$
-\operatorname{div}\left(a_{\varepsilon} \nabla u_{\varepsilon}\right)=f_{\varepsilon},
$$

with the same oscillating coefficients as before but with a varying source term

$$
f_{\varepsilon}(x)=\int_{\varepsilon^{-1} Y} \hat{f}(\xi) \phi_{1}\left(\frac{x}{\varepsilon}, \varepsilon \xi\right) e^{2 \pi i x \cdot \xi} d \xi
$$

where $\phi_{1}(y, \eta)$ is the Bloch first eigenfunction and $\hat{f}(\xi)$ is the Fourier transform of a function $f(x) \in L^{2}\left(\mathbb{R}^{d}\right)$

$$
f(x)=\int_{\mathbb{R}^{d}} \hat{f}(\xi) e^{2 \pi i x \cdot \xi} d \xi
$$

As shown in [10], [11] $f_{\varepsilon}(x)$ converges almost everywhere to $f(x)$ in $\mathbb{R}^{d}$ but assuming that $\hat{f}(\xi)$ decays enough at infinity, using the following Taylor expansion

$$
\phi_{1}(y, \varepsilon \xi)=1+\varepsilon \xi \cdot \nabla_{\eta} \phi_{1}(y, 0)+\frac{1}{2} \varepsilon^{2} \nabla_{\eta} \nabla_{\eta} \phi_{1}(y, 0) \xi \cdot \xi+O\left(\varepsilon^{3}\right),
$$

and Remark 3.2, a more precise asymptotic expansion is

$$
f_{\varepsilon}(x)=f(x)-\varepsilon \chi^{(1)}\left(\frac{x}{\varepsilon}\right) \cdot \nabla f(x)+\varepsilon^{2} \chi^{(2)}\left(\frac{x}{\varepsilon}\right) \cdot \nabla \nabla f(x)+O\left(\varepsilon^{3}\right),
$$

where $\chi^{(1)}(y)$ is the vector of components $\chi_{j}(y)$, defined by (2.6), and $\chi^{(2)}(y)$ is the symmetric matrix of entries $\chi_{k l}(y)$, defined by (2.12). For such a "well prepared" right hand side, equation (5.1) has an explicit solution in terms of Bloch waves

$$
u_{\varepsilon}(x)=\int_{\varepsilon^{-1} Y} \frac{\hat{f}(\xi)}{\varepsilon^{-2} \lambda_{1}(\varepsilon \xi)} \phi_{1}\left(\frac{x}{\varepsilon}, \varepsilon \xi\right) e^{2 \pi i x \cdot \xi} d \xi .
$$

The limit $u$ of $u_{\varepsilon}$ is clearly identified by using Theorem 3.1 which implies that $\varepsilon^{-2} \lambda_{1}(\varepsilon \xi)=$ $4 \pi^{2} a^{*} \xi \cdot \xi+O\left(\varepsilon^{2}\right)$ and thus the Fourier transform of $u(x)$ is $\hat{u}(\xi)=\frac{\hat{f}(\xi)}{4 \pi^{2} a^{*} \xi \cdot \xi}$ which implies that $u$ is the solution of the homogenized equation (2.7).

Of course, a better approximation of $u_{\varepsilon}$ can be obtained by combining the Taylor expansions (3.2) for the eigenvalue $\lambda_{1}$ and (5.4) for the eigenfunction $\phi_{1}$. To explain this improved result we first introduce the following definition.

Definition 5.1. In the context of the Bloch wave method, the "high order homogenized equation" is

$$
-\operatorname{div}\left(a^{*} \nabla v_{\varepsilon}\right)+\varepsilon^{2} \mathbb{D}^{*} \cdot \nabla^{4} v_{\varepsilon}=f .
$$


Remark that (5.7) is similar to (2.27) if one replaces the tensor $\mathbb{D}^{*}$ by $-\mathbb{B}^{*}$ (which is different). By virtue of (3.2), the Fourier transform of the solution $v_{\varepsilon}(x)$ of (5.7) satisfies

$$
\hat{v}_{\varepsilon}(\xi)=\frac{\hat{f}(\xi)}{4 \pi^{2} a^{*} \xi \cdot \xi+\varepsilon^{2}(2 \pi)^{4} \mathbb{D}^{*} \cdot(\xi \otimes \xi \otimes \xi \otimes \xi)}=\frac{\hat{f}(\xi)}{\varepsilon^{-2} \lambda_{1}(\varepsilon \xi)}+O\left(\varepsilon^{4}\right) .
$$

Then, replacing $\phi_{1}$ by its Taylor expansion (5.4) and $\frac{\hat{f}(\xi)}{\varepsilon^{-2} \lambda_{1}(\varepsilon \xi)}$ by (5.8), formula (5.6) becomes formally, by applying the inverse Fourier transform,

$$
u_{\varepsilon}(x)=v_{\varepsilon}(x)-\varepsilon \chi^{(1)}\left(\frac{x}{\varepsilon}\right) \cdot \nabla v_{\varepsilon}(x)+\varepsilon^{2} \chi^{(2)}\left(\frac{x}{\varepsilon}\right) \cdot \nabla \nabla v_{\varepsilon}(x)+O\left(\varepsilon^{3}\right),
$$

which is a high order approximation, much better than just the homogenized solution $u$. Remark that (5.9) is similar to a truncated version of the ansatz (2.25), due to [6] in the context of the two-scale asymptotic expansion method.

Note that the approximation (5.9) is very formal at this stage since the "high order homogenized equation" (5.7) is not well-posed because $\mathbb{D}^{*}$ is non-positive by Theorem 4.1. Nevertheless, the above formal discussion is useful to understand the role of $\mathbb{D}^{*}$ and make a comparison with $\mathbb{B}^{*}$ as follows.

We now switch to the method of two-scale asymptotic expansion. We first rewrite the "high order" homogenized equation (2.27) using formula (4.1), $\mathbb{B}^{*}=-\mathbb{D}^{*}-a^{*} \otimes d^{*}$, which implies

$$
-\operatorname{div}\left(a^{*} \nabla v_{\varepsilon}\right)+\varepsilon^{2} \mathbb{D}^{*} \nabla^{4} v_{\varepsilon}+\varepsilon^{2} \operatorname{div}\left(d^{*} \nabla\left[\operatorname{div}\left(a^{*} \nabla v_{\varepsilon}\right)\right]\right)=f+O\left(\varepsilon^{4}\right) .
$$

Using its truncation $-\operatorname{div}\left(a^{*} \nabla v_{\varepsilon}\right)=f+O\left(\varepsilon^{2}\right)$, we deduce

$$
-\operatorname{div}\left(a^{*} \nabla v_{\varepsilon}\right)+\varepsilon^{2} \mathbb{D}^{*} \nabla^{4} v_{\varepsilon}=f+\varepsilon^{2} \operatorname{div}\left(d^{*} \nabla f\right)+O\left(\varepsilon^{4}\right),
$$

which shares the same differential operator as (5.7) but has a different right hand side.

We now explain the difference between (2.27), or its equivalent version (5.10), and the "high order" homogenized equation (5.7) from the Bloch wave method. The main point is that the previous Bloch wave method was not considering a fixed right hand side $f(x)$ but rather its well prepared version $f_{\varepsilon}$, defined by (5.2). Therefore, to make a fair comparison we have to re-do the analysis of Section 2 but with $f_{\varepsilon}$ instead of $f$. We now outline the only changes to take into account. The first equations in the cascade of equations (2.3), namely those defining $u_{0}, u_{1}$ and $u_{2}$ do not change.

The first change happens with the equation for $u_{3}$ which now is

$$
A_{y y} u_{3}^{\text {new }}+A_{x y} u_{2}+A_{x x} u_{1}=-\chi^{(1)}(y) \cdot \nabla f(x) .
$$

We denote by $u_{3}^{\text {new }}$ the "new" solution of $(5.11)$ and by $u_{3}$ the previous solution, i.e. that with a zero right hand side, defined by (2.18). Since $\chi^{(1)}=\left(\chi_{j}\right)_{1 \leq j \leq d}$ has zero average on $Y$, the compatibility condition of the Fredholm lemma 2.1 is satisfied and the deduced equation (2.13) for $\tilde{u}_{1}$ is unchanged. The new solution is therefore obtained from the previous one by adding a new term due to the right hand side in (5.11)

$$
u_{3}^{\text {new }}(x, y)=u_{3}(x, y)-w(y) \cdot \nabla f(x),
$$

where $w=\left(w_{j}\right)_{1 \leq j \leq d}$ is the solution of (4.4).

The next change occurs in the equation for $u_{4}$ which now is

$$
A_{y y} u_{4}^{\text {new }}+A_{x y} u_{3}^{\text {new }}+A_{x x} u_{2}=\chi^{(2)}(y) \cdot \nabla \nabla f(x) .
$$


The right hand side in (5.13) does not contribute to the compatibility condition of the Fredholm lemma 2.1 since $\chi^{(2)}(y)$ has zero average in $Y$. However the additional term $-w(y) \cdot \nabla f(x)$ in $u_{3}^{\text {new }}$ gives an extra contribution in equation $(2.20)$ for its new solution $\tilde{u}_{2}^{\text {new }}$ which is now

$$
-\operatorname{div}_{x}\left(a^{*} \nabla_{x} \tilde{u}_{2}^{\text {new }}\right)=\mathbb{B}^{*} \nabla_{x}^{4} u+\operatorname{div}_{x} \int_{Y} a(y) \nabla_{y}\left(w(y) \cdot \nabla_{x} f(x)\right) d y .
$$

From (4.6) we know that $\int_{Y} a \nabla_{y} w_{i} \cdot e_{j} d y=\int_{Y} \chi_{i} \chi_{j} d y=d_{i j}^{*}$, which implies that the last equation can be rewritten as

$$
-\operatorname{div}_{x}\left(a^{*} \nabla_{x} \tilde{u}_{2}^{\text {new }}\right)=\mathbb{B}^{*} \nabla_{x}^{4} u+\operatorname{div}_{x}\left(d^{*} \nabla_{x} f\right) .
$$

Replacing $f(x)$ by $-\operatorname{div}_{x}\left(a^{*} \nabla_{x} u\right)$ and recalling (4.5) yields

$$
-\operatorname{div}_{x}\left(a^{*} \nabla_{x} \tilde{u}_{2}^{\text {new }}\right)=\mathbb{B}^{*} \nabla_{x}^{4} u-\left(\mathbb{B}^{*}+\mathbb{D}^{*}\right) \nabla_{x}^{4} u=-\mathbb{D}^{*} \nabla_{x}^{4} u .
$$

Multiplying (5.15) by $\varepsilon^{2}$ and adding it to the homogenized equation (2.7) leads to

$$
-\operatorname{div}_{x}\left(a^{*} \nabla_{x}\left(u+\varepsilon^{2} \tilde{u}_{2}^{\text {new }}\right)\right)+\varepsilon^{2} \mathbb{D}^{*} \nabla_{x}^{4}\left(u+\varepsilon^{2} \tilde{u}_{2}^{\text {new }}\right)=f+O\left(\varepsilon^{4}\right),
$$

which is precisely the "high order" homogenized equation (5.7) for $v_{\varepsilon}$, up to a small $O\left(\varepsilon^{4}\right)$ remainder. Therefore, the two methods agree if one considers the proper right hand sides for the $\varepsilon$-equation. Note that (5.16) can be deduced, not only for the right hand side $f_{\varepsilon}$, but for its first-order approximation $f(x)-\varepsilon \chi^{(1)}\left(\frac{x}{\varepsilon}\right) \cdot \nabla f(x)$ since the second-order term in (5.5) does not contribute to the compatibility condition of (5.13) and higher order terms are negligible. In other words, we have just proved the following result.

Proposition 5.2. The "high order" homogenized equation (5.7), obtained by the Bloch wave method, is equivalent to that, (2.27) or (5.10), obtained by the two-scale asymptotic expansion method if, in the latter case, the right hand side $f$ is replaced by its corrected version

$$
f_{\varepsilon}(x)=f(x)-\varepsilon \chi^{(1)}\left(\frac{x}{\varepsilon}\right) \cdot \nabla f(x) .
$$

Remark 5.3. The high order homogenized equations (5.7) and (5.10) are not well posed since, by virtue of Proposition 3.4, the tensor $\mathbb{D}^{*}$ has the "wrong" sign (the bilinear form associated to the operator $\mathbb{D}^{*} \nabla_{x}^{4}$ is non-positive). Similarly, the other higher order homogenized equation (2.27) is not well posed since its fourth tensor $-\mathbb{B}^{*}$ is not positive too. We now explain how to modify these equations in order to make them well-posed by using a classical Boussinesq trick (see e.g. [9] for historical references ; the same trick has been applied in recent works [17], [19], [16], [1], [2]). The Boussinesq trick is also well known in the study of continuum limits of discrete spring-mass lattices [20]. The key point is that both equations (5.7) and (2.27) are actually defined, up to the addition of a small remainder term of order $\varepsilon^{4}$ : therefore one can modify them adding any term of the same order $\varepsilon^{4}$, without altering their approximate validity. Let us explain the Boussinesq trick for (5.7) (the case of (2.27) is completely similar). We define the minimum value

$$
m=\min _{|\xi|=1} \frac{\mathbb{D}^{*} \cdot(\xi \otimes \xi \otimes \xi \otimes \xi)}{a^{*} \xi \cdot \xi},
$$

which is a non-positive number $m \leq 0$ because of Proposition 3.4 (if $m>0$ were positive, (5.7) is well posed and there is nothing to do). Introducing the non-negative second order tensor $C=-m I d \geq 0$, we define a fourth order tensor $\mathfrak{D}^{*}$ by

$$
\mathfrak{D}^{*} \cdot(\xi \otimes \xi \otimes \xi \otimes \xi)=\mathbb{D}^{*} \cdot(\xi \otimes \xi \otimes \xi \otimes \xi)+\left(a^{*} \xi \cdot \xi\right)(C \xi \cdot \xi) \geq 0 \forall \xi \in \mathbb{R}^{d},
$$


which is non-negative in view of (5.17). Then, the Fourier transform of (5.7)

$$
4 \pi^{2}\left(a^{*} \xi \cdot \xi\right) \hat{v}_{\varepsilon}(\xi)+\varepsilon^{2}(2 \pi)^{4} \mathbb{D}^{*} \cdot(\xi \otimes \xi \otimes \xi \otimes \xi) \hat{v}_{\varepsilon}(\xi)=\hat{f}(\xi)
$$

can be replaced by

$$
4 \pi^{2}\left(a^{*} \xi \cdot \xi\right) \hat{v}_{\varepsilon}(\xi)+\varepsilon^{2}(2 \pi)^{4} \mathfrak{D}^{*} \cdot(\xi \otimes \xi \otimes \xi \otimes \xi) \hat{v}_{\varepsilon}(\xi)=\hat{f}(\xi)+\varepsilon^{2} 4 \pi^{2}(C \xi \cdot \xi) \hat{f}(\xi)+O\left(\varepsilon^{4}\right),
$$

since truncating (5.19) implies $4 \pi^{2}\left(a^{*} \xi \cdot \xi\right) \hat{v}_{\varepsilon}(\xi)=\hat{f}(\xi)+O\left(\varepsilon^{2}\right)$. By the inverse Fourier transform we deduce a well-posed equation which is equivalent to the higher order homogenized equation (5.7), up to order $\varepsilon^{4}$,

$$
-\operatorname{div}\left(a^{*} \nabla v_{\varepsilon}\right)+\varepsilon^{2} \mathfrak{D}^{*} \cdot \nabla^{4} v_{\varepsilon}=f-\varepsilon^{2} \operatorname{div}(C \nabla f)+O\left(\varepsilon^{4}\right) .
$$

From a numerical point of view, (5.20) should be solved (without the $O\left(\varepsilon^{4}\right)$ error term) rather than the ill-posed original equation (5.7). Of course, any choice of matrix $C$, which makes (5.18) non-negative, is acceptable. Therefore, there is a whole family of higher order homogenized equation (5.20), all of them being equivalent up to order $\varepsilon^{4}$. In this context, the dispersive effect is measured by the fourth-order tensor $\mathbb{D}^{*}$ and not by $\mathfrak{D}^{*}$ alone.

In 1-d, since $\mathbb{B}^{*}=0$, we have $\mathbb{D}^{*}=-\mathbb{E}^{*}=-a^{*} d^{*}$ with $d^{*}>0$. Therefore, in 1 - $d$ it is possible to choose $\mathfrak{D}^{*}=0$ and $C=d^{*}$.

Remark 5.4. This entire section was written in a formal way for the ease of presentation. In particular, we never explained in which sense the remainder terms $O\left(\varepsilon^{k}\right)$ have to be understood. Rigorous results about the better approximation properties of the high order homogenized equation (5.7) have first been established in [19], [16], in the context of the wave equation.

\section{Application to wave propagation}

The above analysis can be extended from the elliptic equation (1.1) to a time dependent setting. Although we could expose the case of a parabolic equation, it makes more sense to consider the wave equation because of the importance of dispersive effects for wave propagation in periodic media (see e.g. [24], [19], [16], [5], [1], [2]). Therefore, for the rest of this section we consider the following wave equation, posed in the entire space $\mathbb{R}^{d}$,

$$
\left\{\begin{array}{l}
\frac{\partial^{2} u_{\varepsilon}}{\partial t^{2}}-\operatorname{div}\left(a_{\varepsilon} \nabla u_{\varepsilon}\right)=f, \\
u_{\varepsilon}(0, x)=u^{\text {init }}(x), \quad \frac{\partial u_{\varepsilon}}{\partial t}(0, x)=v^{\text {init }}(x),
\end{array}\right.
$$

with periodic coefficients $a_{\varepsilon}(x):=a\left(\frac{x}{\varepsilon}\right)$, a right hand side $f(t, x)$ and initial date $u^{\text {init }}(x), v^{\text {init }}(x)$.

Two-scale asymptotic expansion method. As is well known, the same asymptotic analysis than in Section 2 can be performed, up to delicate corrector issues for which the reader is referred to [8]. We only sketch the differences with the analysis of the elliptic case. The two-scale ansatz for the solution of $(6.1)$ is

$$
u_{\varepsilon}(t, x)=\sum_{n=0}^{\infty} \varepsilon^{n} u_{n}\left(t, x, \frac{x}{\varepsilon}\right)
$$


where $u_{n}(t, x, \cdot)$ are $Y$-periodic and satisfy the cascade of equations

$$
\left\{\begin{array}{cl}
\operatorname{order} \varepsilon^{-2}, & 0=A_{y y} u_{0} \\
\operatorname{order} \varepsilon^{-1}, & 0=A_{y y} u_{1}+A_{x y} u_{0} \\
\operatorname{order} \varepsilon^{0}, & f=A_{y y} u_{2}+A_{x y} u_{1}+A_{x x} u_{0}+\frac{\partial^{2} u_{0}}{\partial t^{2}} \\
\operatorname{order} \varepsilon^{1}, & 0=A_{y y} u_{3}+A_{x y} u_{2}+A_{x x} u_{1}+\frac{\partial^{2} u_{1}}{\partial t^{2}} \\
& \vdots \\
\operatorname{order} \varepsilon^{n}, & 0=A_{y y} u_{n+2}+A_{x y} u_{n+1}+A_{x x} u_{n}+\frac{\partial^{2} u_{n}}{\partial t^{2}} \\
& \vdots
\end{array}\right.
$$

The equations for $u_{0}$ and $u_{1}$ do not change and they yield

$$
u_{0}(t, x, y)=u(t, x) \quad \text { and } \quad u_{1}(t, x, y)=-\chi_{i}(y) \frac{\partial u}{\partial x_{i}}(t, x)+\tilde{u}_{1}(t, x) .
$$

An additional time derivative appears in all the higher order equations of (6.2). The compatibility condition (Fredholm alternative) of the equation for $u_{2}$ leads to the homogenized equation

$$
\frac{\partial^{2} u}{\partial t^{2}}-\operatorname{div}\left(a^{*} \nabla u\right)=f
$$

with the same homogenized matrix $a^{*}$ defined by (2.8). Then, $u_{2}$ is given by

$$
u_{2}(t, x, y)=\chi_{i j}(y) \frac{\partial^{2} u}{\partial x_{i} x_{j}}(t, x)-\chi_{i}(y) \frac{\partial \tilde{u}_{1}}{\partial x_{i}}(t, x)+\tilde{u}_{2}(t, x)
$$

with the same functions $\chi_{i j}$ defined by (2.12). The equation for $u_{3}$ becomes

$$
A_{y y} u_{3}=-\frac{\partial^{2} \tilde{u}_{1}}{\partial t^{2}}(t, x)+\chi_{i}(y) \frac{\partial^{3} u}{\partial t^{2} \partial x_{i}}(t, x)-A_{x y} u_{2}-A_{x x} u_{1} .
$$

Since $\chi_{i}$ has zero-average, the compatibility condition of (6.5) leads to an equation, similar to (2.13),

$$
\frac{\partial^{2} \tilde{u}_{1}}{\partial t^{2}}-\operatorname{div}\left(a^{*} \nabla \tilde{u}_{1}\right)=C^{*} \nabla^{3} u=0,
$$

with the same vanishing tensor $C^{*}$, defined by (2.14). From this we deduce a new formula for $u_{3}$, where the first term (the only new one, compared to (2.18)) is due to the source term proportional to $\chi_{i}$ in $(6.5)$, namely

$$
\begin{aligned}
u_{3}(t, x, y)= & w_{i}(y) \frac{\partial^{3} u}{\partial x_{i} \partial t^{2}}(t, x)+\chi_{i j k}(y) \frac{\partial^{3} u}{\partial x_{i} \partial x_{j} \partial x_{k}}(t, x) \\
& +\chi_{i j}(y) \frac{\partial^{2} \tilde{u}_{1}}{\partial x_{i} \partial x_{j}}(t, x)-\chi_{i}(y) \frac{\partial \tilde{u}_{2}}{\partial x_{i}}(t, x)+\tilde{u}_{3}(t, x),
\end{aligned}
$$

where the functions $w_{i}$ and $\chi_{i j k}$ are still defined by (4.4) and (2.19), respectively. Plugging (6.7) in the right hand side of the equation for $u_{4}$, its compatibility condition gives an equation for $\tilde{u}_{2}$ which is similar to (2.22) except for an additional term due to the new term $w_{i} \frac{\partial^{3} u}{\partial x_{i} \partial t^{2}}$ in $u_{3}$. More precisely we get

$$
\frac{\partial^{2} \tilde{u}_{2}}{\partial t^{2}}-\operatorname{div}\left(a^{*} \nabla \tilde{u}_{2}\right)=\mathbb{B}^{*} \nabla^{4} u+\operatorname{div}\left(\frac{\partial^{3} u}{\partial x_{i} \partial t^{2}} \int_{Y} a \nabla_{y} w_{i} d y\right),
$$


where $\mathbb{B}^{*}$ is defined by (2.21). From (4.6) we know that $\int_{Y} a \nabla_{y} w_{i} \cdot e_{j} d y=\int_{Y} \chi_{i} \chi_{j} d y=d_{i j}^{*}$, and (4.1) states that $\mathbb{D}^{*}=-\mathbb{B}^{*}-a^{*} \otimes d^{*}$. Thus the last term in (6.8) simplifies

$$
\operatorname{div}\left(\frac{\partial^{3} u}{\partial x_{i} \partial t^{2}} \int_{Y} a \nabla_{y} w_{i} d y\right)=\operatorname{div}\left(d^{*} \nabla \frac{\partial^{2} u}{\partial t^{2}}\right)
$$

Using the homogenized equation (6.3), i.e. replacing $\frac{\partial^{2} u}{\partial t^{2}}$ by $f+\operatorname{div}\left(a^{*} \nabla u\right)$, we deduce that (6.8) is equivalent to

$$
\frac{\partial^{2} \tilde{u}_{2}}{\partial t^{2}}-\operatorname{div}\left(a^{*} \nabla \tilde{u}_{2}\right)=-\mathbb{D}^{*} \nabla^{4} u+\operatorname{div}\left(d^{*} \nabla f\right),
$$

from which we can obtain the higher order homogenized equation for the function $v_{\varepsilon}(t, x)=$ $u(t, x)+\varepsilon^{2} \tilde{u}_{2}(t, x)$, that is

$$
\frac{\partial^{2} v_{\varepsilon}}{\partial t^{2}}-\operatorname{div}\left(a^{*} \nabla v_{\varepsilon}\right)+\varepsilon^{2} \mathbb{D}^{*} \nabla^{4} v_{\varepsilon}=f+\varepsilon^{2} \operatorname{div}\left(d^{*} \nabla f\right)+O\left(\varepsilon^{4}\right) .
$$

Note that the initial data did not enter the entire asymptotic process which is, of course, purely formal at this stage. The fourth-order tensor in (6.9) is $\mathbb{D}^{*}$, as given by the Bloch wave method, and not $\mathbb{B}^{*}$ as in the previous elliptic case, see (2.27). Nevertheless, in the elliptic case, we also recover the tensor $\mathbb{D}^{*}$ in $(5.10)$ but this is at the price of a manipulation of the equation (replacing $\operatorname{div}\left(a^{*} \nabla v_{\varepsilon}\right)$ by $-f$ in some terms). Here, in the hyperbolic case, we directly obtain $\mathbb{D}^{*}$ without any appearance of $\mathbb{B}^{*}$. In both cases, elliptic and hyperbolic, we obtain a modification of the source term: see the same right hand sides in (5.10) and (6.9).

Bloch-wave expansion method: Let us replace the fixed (with respect to $\varepsilon$ ) initial data and source term in (6.1) by well-prepared initial data and force in terms of Bloch waves. Denoting by $\hat{u}^{\text {init }}(\xi), \hat{v}^{\text {init }}(\xi)$ and $\hat{f}(t, \xi)$ the Fourier transforms of $u^{\text {init }}(x), v^{\text {init }}(x)$ and $f(t, x)$ (in the sense of $(5.3))$, we introduce these new forcing term and initial data

$$
\begin{gathered}
f_{\varepsilon}(t, x)=\int_{\varepsilon^{-1} Y} \hat{f}(t, \xi) \phi_{1}\left(\frac{x}{\varepsilon}, \varepsilon \xi\right) e^{2 \pi i x \cdot \xi} d \xi, \\
u_{\varepsilon}^{\text {init }}(x)=\int_{\varepsilon^{-1} Y} \hat{u}^{\text {init }}(\xi) \phi_{1}\left(\frac{x}{\varepsilon}, \varepsilon \xi\right) e^{2 \pi i x \cdot \xi} d \xi, \quad v_{\varepsilon}^{\text {init }}(x)=\int_{\varepsilon^{-1} Y} \hat{v}^{\text {init }}(\xi) \phi_{1}\left(\frac{x}{\varepsilon}, \varepsilon \xi\right) e^{2 \pi i x \cdot \xi} d \xi,
\end{gathered}
$$

and change (6.1) into

$$
\left\{\begin{array}{l}
\frac{\partial^{2} u_{\varepsilon}}{\partial t^{2}}-\operatorname{div}\left(a_{\varepsilon} \nabla u_{\varepsilon}\right)=f_{\varepsilon}(t, x), \\
u_{\varepsilon}(0, x)=u_{\varepsilon}^{\text {init }}(x), \quad \frac{\partial u_{\varepsilon}}{\partial t}(0, x)=v_{\varepsilon}^{\text {init }}(x) .
\end{array}\right.
$$

Using Lemma 3.3 the solution of (6.10) is given by

$$
u_{\varepsilon}(t, x)=\int_{\varepsilon^{-1} Y} \hat{u}_{1}^{\varepsilon}(t, \xi) \phi_{1}\left(\frac{x}{\varepsilon}, \varepsilon \xi\right) e^{2 \pi i x \cdot \xi} d \xi
$$

where, for any $\xi \in \varepsilon^{-1} Y, \hat{u}_{1}^{\varepsilon}(t, \xi)$ is a solution of the following ordinary differential equation

$$
\left\{\begin{array}{l}
\frac{d^{2} \hat{u}_{1}^{\varepsilon}}{d t^{2}}+\varepsilon^{-2} \lambda_{1}(\varepsilon \xi) \hat{u}_{1}^{\varepsilon}=\hat{f}(t, \xi), \\
\hat{u}_{1}^{\varepsilon}(0, \xi)=\hat{u}^{\text {init }}(\xi), \quad \frac{d \hat{u}_{1}^{\varepsilon}}{d t}(0, \xi)=\hat{v}^{\text {init }}(\xi) .
\end{array}\right.
$$


Using the Taylor expansion (3.2) of $\lambda_{1}$, we deduce that

$$
\left\{\begin{array}{l}
\frac{d^{2} \hat{u}_{1}^{\varepsilon}}{d t^{2}}+\left(4 \pi^{2} a^{*} \xi \cdot \xi+\varepsilon^{2}(2 \pi)^{4} \mathbb{D}^{*} \cdot(\xi \otimes \xi \otimes \xi \otimes \xi)\right) \hat{u}_{1}^{\varepsilon}=\hat{f}(t, \xi)+O\left(\varepsilon^{4}\right), \\
\hat{u}_{1}^{\varepsilon}(0, \xi)=\hat{u}^{\text {init }}(\xi), \quad \frac{d \hat{u}_{1}^{\varepsilon}}{d t}(0, \xi)=\hat{v}^{\text {init }}(\xi) .
\end{array}\right.
$$

At least formally, dropping the $O\left(\varepsilon^{4}\right)$ in the above equation, $\hat{u}_{1}^{\varepsilon}(t, \xi)$ is well approximated by $\hat{v}_{\varepsilon}(t, \xi)$ which is the Fourier transform of the solution $v_{\varepsilon}(t, x)$ of the following high order homogenized equation

$$
\left\{\begin{array}{l}
\frac{\partial^{2} v_{\varepsilon}}{\partial t^{2}}-\operatorname{div}\left(a^{*} \nabla v_{\varepsilon}\right)+\varepsilon^{2} \mathbb{D}^{*} \cdot \nabla_{x}^{4} v_{\varepsilon}=f(t, x) \\
v_{\varepsilon}(0, x)=u^{\text {init }}(x), \quad \frac{\partial v_{\varepsilon}}{\partial t}(0, x)=v^{\text {init }}(x)
\end{array}\right.
$$

Contrary to the elliptic case, the left hand side operator in (6.14) is identical to that in (6.9) which was obtained by two-scale asymptotic expansions. Only the right hand side differs, as was already explained in Section 5. As stated in Proposition 6.1, the right hand side of (6.9) can be made equal to $f$ if the source term is corrected or "well prepared" in the wave equation (6.1). We summarize our results in the following proposition.

Proposition 6.1. The "high order" homogenized equation of the two-scale asymptotic expansion method applied to the wave equation (6.1) is

$$
\frac{\partial^{2} v_{\varepsilon}}{\partial t^{2}}-\operatorname{div}\left(a^{*} \nabla v_{\varepsilon}\right)+\varepsilon^{2} \mathbb{D}^{*} \nabla^{4} v_{\varepsilon}=f+\varepsilon^{2} \operatorname{div}\left(d^{*} \nabla f\right) .
$$

On the other hand, the "high order" homogenized equation obtained by the Bloch wave method is

$$
\frac{\partial^{2} v_{\varepsilon}}{\partial t^{2}}-\operatorname{div}\left(a^{*} \nabla v_{\varepsilon}\right)+\varepsilon^{2} \mathbb{D}^{*} \nabla^{4} v_{\varepsilon}=f
$$

The latter can also be obtained by the method of two-scale asymptotic expansion if, in (6.1), the source term $f(t, x)$ is replaced by its corrected version

$$
f_{\varepsilon}(t, x)=f(t, x)-\varepsilon \chi^{(1)}\left(\frac{x}{\varepsilon}\right) \cdot \nabla f(t, x) .
$$

Of course, as already remarked in Section 5, problem (6.14) is ill-posed because $\mathbb{D}^{*}$ has the wrong sign. However, the same Boussinesq argument of Remark 5.3 works here and the decomposition (5.18) allows us to transform (6.13) in

$$
\left\{\begin{array}{c}
\left(1+\varepsilon^{2} 4 \pi^{2} C \xi \cdot \xi\right) \frac{d^{2} \hat{u}_{1}^{\varepsilon}}{d t^{2}}+\left(4 \pi^{2} a^{*} \xi \cdot \xi+\varepsilon^{2}(2 \pi)^{4} \mathfrak{D}^{*} \cdot(\xi \otimes \xi \otimes \xi \otimes \xi)\right) \hat{u}_{1}^{\varepsilon} \\
=\hat{f}(t, \xi)+\varepsilon^{2} 4 \pi^{2}(C \xi \cdot \xi) \hat{f}(t, \xi)+O\left(\varepsilon^{4}\right), \\
\hat{u}_{1}^{\varepsilon}(0, \xi)=\hat{u}^{\text {init }}(\xi), \quad \frac{d \hat{u}_{1}^{\varepsilon}}{d t}(0, \xi)=\hat{v}^{\text {init }}(\xi),
\end{array}\right.
$$

which is the Fourier transform of the well-posed problem (because $C$ and $\mathfrak{D}^{*}$ are non-negative)

$$
\left\{\begin{array}{c}
\frac{\partial^{2} v_{\varepsilon}}{\partial t^{2}}-\varepsilon^{2} \operatorname{div}\left(C \nabla \frac{\partial^{2} v_{\varepsilon}}{\partial t^{2}}\right)-\operatorname{div}\left(a^{*} \nabla v_{\varepsilon}\right)+\varepsilon^{2} \mathfrak{D}^{*} \cdot \nabla^{4} v_{\varepsilon} \\
=f(t, x)-\varepsilon^{2} \operatorname{div}(C \nabla f(t, x))+O\left(\varepsilon^{4}\right), \\
v_{\varepsilon}(0, x)=u^{\text {init }}(x), \quad \frac{\partial v_{\varepsilon}}{\partial t}(0, x)=v^{\text {init }}(x) .
\end{array}\right.
$$


In other words, (6.16) is a stabilized version of (6.14) which does not sacrifice accuracy, up to order $O\left(\varepsilon^{4}\right)$. In numerical practice, system (6.16) should be solved instead of (6.14).

It was proved in [19], [16] (see also [2]) that the solution $v_{\varepsilon}$ of (6.16) provides an approximation of the exact solution $u_{\varepsilon}$ of (6.1), up to an error term of order $\varepsilon$ in the $L_{t}^{\infty}\left(L_{x}^{2}\right)$-norm for long times up to $T \varepsilon^{-2}$.

Acknowledgments This research was partially supported by the Indo-French Centre for Applied Mathematics (IFCAM). G. A. is a member of the DEFI project at INRIA Saclay Ile-deFrance.

\section{References}

[1] A. Abdulle, M. Grote, Ch. Stohrer: Finite element heterogeneous multiscale method for the wave equation: long-time effects, Multiscale Model. Simul. 12 (2014), pp.1230-1257.

[2] A. Abdulle \& T. N. Pouchon: A priori error analysis of the finite element heterogeneous multiscale method for the wave equation in heterogeneous media over long time, preprint 2015.

[3] A. Abdulle \& T. N. Pouchon: Effective models for the multidimensional wave equation in heterogeneous media over long time, preprint 2016.

[4] G. Allaire: Shape Optimization by the Homogenization Method, Applied Mathematical Sciences 146, Springer-Verlag, New-York, 2002, 456 pp.

[5] G. Allaire, M. Palombaro, J. Rauch: Diffractive Geometric Optics for Bloch Wave Packets, Archive Rat. Mech. Anal., 202, pp.373-426 (2011).

[6] N. Bakhvalov, G. Panasenko: Homogenization: Averaging Processes in Periodic Media, Kluwer, Dordrecht (1989).

[7] A. Bensoussan, J.-L. Lions \& G. Papanicolaou: Asymptotic analysis for periodic structures, corrected reprint of the 1978 original, AMS Chelsea Publishing, Providence, RI, 2011, 398 pp.

[8] S. Brahim-Otsmane, G. Francfort \& F. Murat: Correctors for the homogenization of the wave and heat equations, J. Math. Pures Appl. (9) 71:197-231 (1992).

[9] C. I. Christov, G. A. Maugin \& M. G. Velarde: Well-posed Boussinesq paradigm with purely spatial higher-order derivatives, Phys. Rev. E, 54, pp.3621-3638 (1996).

[10] C. Conca And M. Vanninathan: Homogenization of periodic structures via Bloch decomposition, SIAM J. Appl. Math., 57 (1997), 1639-1659.

[11] C. Conca, R. Orive \& M. Vanninathan: Bloch approximation in homogenization and applications, SIAM J. Math. Anal., 33 (5) (2002), 1166-1198.

[12] C. Conca, R. Orive \& M. Vanninathan: "On Burnett coefficients in periodic media", J. Math. Phys., 47 (3) (2006), 11 pp.

[13] C. Conca, J. San Martín, L. Smaranda \& M. Vanninathan: "On Burnett coefficients in periodic media in low contrast regime", J. Math. Phys., 49 (5) (2008), 23 pp. 
[14] C. Conca, J. San Martín, L. Smaranda \& M. Vanninathan: "Optimal bounds on dispersion coefficient in one-dimensional periodic media", Math. Models Methods Appl. Sci., 19 (9) (2009), 1743-1764.

[15] C. Conca, J. San Martín, L. Smaranda \& M. Vanninathan: "Burnett coefficients and laminates", Appl. Anal., 91 (6) (2012), 1155-1176.

[16] T. Dohnal, A. Lamacz \& B. Schweizer: "Bloch-wave homogenization on large time scales and dispersive effective wave equations", Multiscale Model. Simul., 12 (2) (2014), 488-513.

[17] J. Fish, W. Chen, \& G. NAGAI: Non-local dispersive model for wave propagation in heterogeneous media: multi-dimensional case, Internat. J. Numer. Methods Engrg., 54(3):347-363, 2002.

[18] S.S. Ganesh, M. Vanninathan: "Bloch wave homogenization of linear elasticity system", ESAIM Control Optim. Calc. Var., 11 (4) (2005), 542-573.

[19] A. LAmacz: "Dispersive effective models for waves in heterogeneous media", Math. Models Methods Appl. Sci., 21 (9) (2011), 1871-1899.

[20] M. Lombardo, H. Askes: Elastic wave dispersion in microstructured membranes, Proc. R. Soc. A (2010) 466, pp.1789-1807.

[21] S. Moskow \& M. Vogelius: "First order corrections to the homogenized eigenvalues of a periodic composite medium. A convergence proof", Proc. Roy. Soc. Edinburg, 127, pp.1263-1295 (1997).

[22] M. Reed, B. Simon: Methods of modern mathematical physics, Academic Press, New York (1978).

[23] E. SANChez-PALEnCIA: Non-Homogeneous Media and Vibration Theory Springer Lecture Notes in Physics 129 (1980).

[24] F. Santosa, W. Symes: "A dispersive effective medium for wave propagation in periodic composites", SIAM J. Appl. Math., 51 (1991), 984-1005.

[25] V. P. Smyshlyaev, K. D. Cherednichenko: "On rigorous derivation of strain gradient effects in the overall behaviour of periodic heterogeneous media", The J. R. Willis 60th anniversary volume. J. Mech. Phys. Solids, 48 (6-7) (2000), 1325-1357.

[26] L. TARTAR: The General Theory of Homogenization: A Personalized Introduction, Lecture Notes of the Unione Matematica Italiana, Springer-Verlag, Berlin Heidelberg 2009, pp. 471.

[27] C. Wilcox: Theory of Bloch waves, J. Anal. Math. 33, pp. 146-167 (1978). 